\title{
An Embedding Approach for the Design of State-Feedback Tracking Controllers for References with Jumps
}

\author{
Ricardo G. Sanfelice, J. J. Benjamin Biemond, Nathan van de Wouw, and W. P. Maurice \\ H. Heemels \\ ${ }^{1}$ Department of Aerospace and Mechanical Engineering, University of Arizona, 1130 N. Mountain Ave, AZ 85721, USA. \\ ${ }^{2,3,4}$ Department of Mechanical Engineering, Eindhoven University of Technology, P.O. Box 513, 5600 MB Eindhoven, \\ The Netherlands.
}

\section{SUMMARY}

We study the problem of designing state-feedback controllers to track time-varying state trajectories that may exhibit jumps. Both plants and controllers considered are modeled as hybrid dynamical systems, which are systems with both continuous and discrete dynamics, given in terms of a flow set, a flow map, a jump set, and a jump map. Using recently developed tools for the study of stability in hybrid systems, we recast the tracking problem as the task of asymptotically stabilizing a set, the tracking set, and derive conditions for the design of state-feedback tracking controllers with the property that the jump times of the plant coincide with those of the given reference trajectories. The resulting tracking controllers guarantee that solutions of the plant starting close to the reference trajectory stay close to it and that the difference between each solution of the controlled plant and the reference trajectory converges to zero asymptotically. Constructive conditions for tracking control design in terms of Linear Matrix Inequalities (LMIs) are proposed for a class of hybrid systems with linear maps and input-triggered jumps. The results are illustrated by various examples. Copyright (c) 2011 John Wiley \& Sons, Ltd.

Received ...

KEY WORDS: Tracking control; hybrid systems; asymptotic stability.

Copyright (c) 2011 John Wiley \& Sons, Ltd.

Prepared using rncauth.cls [Version: 2010/03/27 v2.00]
This is the pre-peer reviewed version of the following article: Sanfelice, R. G.; Biemond, J. J. B.; van de Wouw, N. \& Heemels, W. P. M. H. An Embedding Approach for the Design of Tracking Controllers for References with Jumps To appear in the International Journal of Robust and Nonlinear Control, 2013. 


\section{INTRODUCTION}

The literature on stability analysis and stabilization of equilibria for systems with state jumps is relatively well developed; see, e.g., [Ye et al.(1998), Lygeros et al.(2003), Goebel and Teel(2006), Sanfelice et al.(2007), Goebel et al.(2009)] for results for hybrid systems; [Pereira and Silva(2004), Brogliato(2004), Leine and van de Wouw(2008)] for results in the scope of measure differential inclusions; [Brogliato(1996), Camlibel et al.(2006)] in the scope of complementarity systems; [Bainov and Simeonov(1989), Lakshmikantham et al.(1989)] in the scope of impulsive dynamical systems; and many others. On the other hand, in many control problems, such as tracking, output regulation, synchronization, and observer design, the goal consists of stabilizing time-varying trajectories. To effectively tackle such problems for hybrid systems, results on the stability and stabilization of time-varying trajectories of such systems are imperative. Unfortunately, general results for stabilizing impulsive/discontinuous, or, more generally, hybrid trajectories are not currently available. Notable specific solutions to stabilization of such trajectories are given by the work in [Menini and Tornambe(2001), Martinelli et al.(2002), Galeani et al.(2008), Galeani et al.(2011)], in which the state estimation and tracking problems for particular classes of mechanical systems with impacts are addressed, the work in [Heemels et al.(2011)], in which an observer-based control design problem for a class of complementarity systems is studied, the work in [Leine and van de Wouw(2008)], in which tracking control and observer design problems for a class of measure differential inclusions is solved, the work in [Bourgeot and Brogliato(2005), Brogliato et al.(1997), Brogliato et al.(2000), Morarescu and Brogliato(2010)], in which a tracking

*Correspondence to: Department of Aerospace and Mechanical Engineering, University of Arizona, 1130 N. Mountain Ave, AZ 85721, USA. Email: sricardo@u.arizona.edu. Research by R. G. Sanfelice has been partially supported by the National Science Foundation under CAREER Grant no. ECS-1150306 and by the Air Force Office of Scientific Research under Grant no. FA9550-12-1-0366. Research by J. J. B. Biemond, N. van de Wouw, and W. P. M. H. Heemels has been partially supported by the Netherlands organization for scientific research (NWO) and the European Union Seventh Framework Programme [FP7/2007-2013] under grant agreement n257462 HYCON2 Network of excellence". 
problem for a class of mechanical systems with unilateral constraints is addressed, and the work in [Sanfelice et al.(2007)] considering the juggling problem as a tracking problem.

In this paper, we present sufficient conditions characterizing controllers solving a state tracking control problem for a general class of hybrid systems. More specifically, we consider plants given in terms of a constrained flow equation ${ }^{\dagger}$

$$
\dot{\xi}=f_{p}(\xi, u) \quad(\xi, u) \in C_{p}
$$

and a constrained jump inclusion

$$
\xi^{+} \in G_{p}(\xi, u) \quad(\xi, u) \in D_{p},
$$

with output function given by the identity, i.e., $y=\xi$. The set $C_{p}$ is the flow set, which is where continuous evolution or flows are possible. The single-valued map $f_{p}$, called the flow map, defines the differential equation governing the flows. The set $D_{p}$ is the jump set, which collects the points from where discrete evolution or jumps are possible. From this set, the state is updated via the set-valued map $G_{p}$, called the jump map. For this class of hybrid systems, a controller assigning the input $u$ and measuring $\xi$ is to be designed such that the difference between $\xi$ and the reference trajectory $r$, which may both flow and jump, is well behaved in a sense to be made more precise later. Without being precise about a notion of tracking at this point, it should be expected that the tracking controller guarantees both stability and attractivity properties relative to the reference trajectory. Stability consists of the property that solutions to the plant starting close to the reference stay close to it while attractivity consists of the property that the distance between the plant's solution component and the reference decreases asymptotically. A major challenge in guaranteeing these properties for hybrid systems is that the jump times of the reference trajectory and the state of the controlled plant do not coincide in general. In Section 2 we discuss in more detail how this complicates the tracking control design problem. The proposed approach in this article consists of recasting the state tracking problem for hybrid systems, which is defined in Section 3, as the

${ }^{\dagger}$ As defined in Section 3, the solutions to (1)-(2) will be given by (absolutely) continuous functions on each interval of flow. 
stabilization of a closed set that embeds the reference trajectory. Exploiting sufficient conditions for asymptotic stability of closed sets for hybrid systems, in Section 4 we present sufficient conditions for a class of hybrid state-feedback tracking controllers enforcing that the jump times of the plant coincide with those of the given reference trajectory. Section 5 considers a special case of hybrid systems with linear maps and jumps triggered by the inputs and proposes constructive conditions for control design given in terms of LMIs. The proposed approach and results are illustrated by examples in Section 6. Section 7 presents concluding remarks.

Notation: The following notational conventions are used throughout the paper. The $n$-dimensional Euclidean space is denoted by $\mathbb{R}^{n}$, real numbers as $\mathbb{R}$, nonnegative real numbers by $\mathbb{R}_{\geq 0}$, and natural numbers including 0 by $\mathbb{N}$. Given a set $S, \bar{S}$ denotes its closure; given a vector $x \in \mathbb{R}^{n},|x|$ denotes the Euclidean vector norm; given a set $S \subset \mathbb{R}^{n}$ and a point $x \in \mathbb{R}^{n}$, we define $|x|_{S}:=\inf _{y \in S}|x-y|$ as the distance from $x$ to the set $S$. Given vectors $x$ and $y$, we write $\left[x^{\top} y^{\top}\right]^{\top}$ with the shorthand notation $(x, y)$. The inner product operation between two vectors $x$ and $y$ is denoted $\langle x, y\rangle$. Given a continuously differentiable function $V, \nabla V$ denotes its gradient. Given a locally Lipschitz function $V, V^{\circ}(x, w)$ denotes the Clarke generalized derivative of $V$ at $x$ in the direction $w$ [Clarke(1990)], i.e., $V^{\circ}(x, w)=\max _{\zeta \in \partial V(x)}\langle\zeta, w\rangle$, where $\partial V(x)$ is the generalized gradient of $V$ in the sense of Clarke, which is a closed, convex, and nonempty set equal to the convex hull of all limit sequences of $\nabla V\left(x_{i}\right)$ with $x_{i} \rightarrow x$ taking value away from every set of measure zero in which $V$ is nondifferentiable. A function $\alpha: \mathbb{R}_{\geq 0} \rightarrow \mathbb{R}_{\geq 0}$ is said to belong to class- $\mathcal{K}(\alpha \in \mathcal{K})$ if it is continuous, zero at zero, and strictly increasing and to belong to class- $\mathcal{K}_{\infty}\left(\alpha \in \mathcal{K}_{\infty}\right)$ if it belongs to class- $\mathcal{K}$ and is unbounded. $\mathcal{P} \mathcal{D}$ denotes the set of real-valued positive definite functions, i.e., $\rho \in \mathcal{P} \mathcal{D}$ implies $\rho(0)=0$ and $\rho(s)>0$ for all $s \neq 0$. The identity function is denoted Id. For a symmetric matrix $A$, we denote by $\lambda_{\min }(A)$ and $\lambda_{\max }(A)$ its minimum and maximum eigenvalue, respectively. 


\section{OBSTACLES TO TRACKING CONTROL DESIGN FOR HYBRID SYSTEMS}

To illustrate the difficulties in tracking control design problems for systems with state jumps, consider a scalar, single-valued hybrid plant as in (1)-(2) with state $\xi$ and output $y=\xi$, and the reference trajectory to be tracked given by the sawtooth signal shown in Figure 1, which has discontinuities when reaching 1 . Trajectories $\xi$ to the plant can be specified as functions defined on hybrid time domains $\operatorname{dom} \xi$, which are subsets of $\mathbb{R}_{\geq 0} \times \mathbb{N}$ and parameterize the trajectories by flow time $t$ and jump time $j$, see [Goebel et al.(2009)]. A common approach used in tracking control of continuous-time and discrete-time plants consists of defining the tracking error and then analyzing the resulting time-varying error dynamics; a different approach to tracking control that is based on dynamic inversion appeared in [Devasia et al.(1996)]. Following the former approach, the reference trajectory $r$ on the hybrid time domain dom $r$ is given by

$$
r(t, j)=t-t_{j}^{r} \quad \forall t \in\left[t_{j}^{r}, t_{j+1}^{r}\right]
$$

where $t_{j}^{r}=j, j \in \mathbb{N}$. Note that $r(t, j) \in[0,1]$ for all $(t, j) \in \operatorname{dom} r$, where $\operatorname{dom} r$ is the union of $\left[t_{j}^{r}, t_{j+1}^{r}\right] \times\{j\}$ for every $j \in \mathbb{N}$. Let

$$
\mathcal{T}_{r}:=\bigcup_{j \in \mathbb{N}>0}\left\{\left(t_{j}^{r}, j-1\right)\right\}
$$

denote the fixed values of $(t, j)$ at which $r$ jumps. Then, the dynamics of the tracking error $e$ defined

as

$$
e=y-r(t, j)=\xi-r(t, j)
$$

are given by the flow equation

$$
\dot{e}=f_{p}(e+r(t, j), u)-1
$$

when

$$
(e+r(t, j), u) \in C_{p} \quad \text { and } \quad t \in\left[t_{j}^{r}, t_{j+1}^{r}\right],
$$

and by the jump equation

$$
e^{+}=G_{e}(e+r(t, j), u, t, j)
$$


when

$$
(e+r(t, j), u) \in D_{p} \quad \text { or } \quad(t, j) \in \mathcal{T}_{r},
$$

where $G_{e}$ is defined at every point satisfying (8) as

$$
G_{e}(e+r, u, t, j)= \begin{cases}G_{p}(e+r, u)-r & (e+r, u) \in D_{p},(t, j) \notin \mathcal{T}_{r} \\ e+r & (e+r, u) \notin D_{p},(t, j) \in \mathcal{T}_{r} \\ G_{p}(e+r, u) & (e+r, u) \in D_{p},(t, j) \in \mathcal{T}_{r},\end{cases}
$$

where $G_{p}$ is the jump map of the plant (1)-(2).

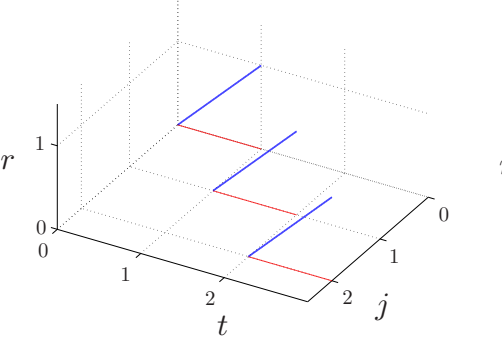

(a) Hybrid arc $r$.

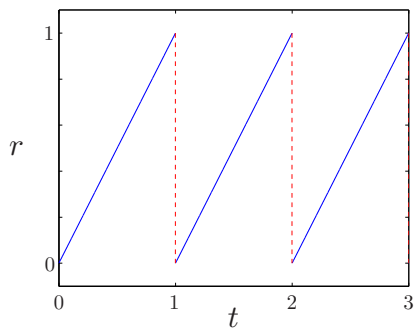

(b) Projection onto $\mathbb{R}_{\geq 0}$.

Figure 1. Reference trajectory for the tracking control problem in Section 2.

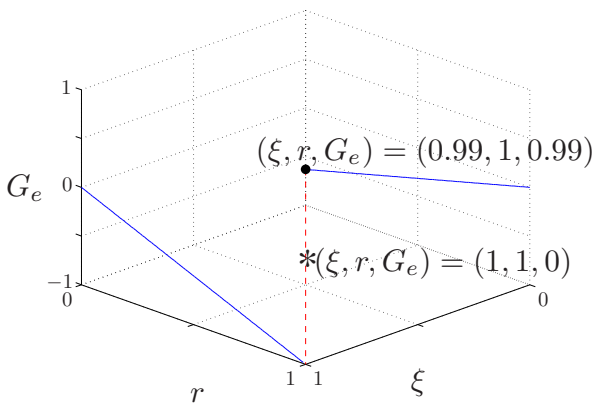

Figure 2. A resulting jump map $G_{e}$ for the error system in the tracking control problem of Section 2 when $G_{p}(\xi, u)=0$ and $D_{p}=\{(\xi, u): \xi=1\}$. The map $G_{e}$ is defined for each $(\xi, r) \in(\mathbb{R} \times\{1\}) \times(\{1\} \times$ $[0,1])$.

A first challenge to tracking control for hybrid systems is due to the impossibility of writing conditions (6) and (8) in terms of the tracking error solely. Note that this issue also emerges in the continuous-time and discrete-time settings, but it is aggravated in hybrid systems due to the 
fact that the flow and jump conditions depend on the state. As a consequence, the dynamics of the tracking error depend on $\xi$ and $r$. Now, suppose that a feedback law $u=\kappa_{c}(y, r)$ is designed to map the error to zero when both the plant's state $\xi$ and $r$ jump simultaneously, that is, the third case in the definition of $G_{e}$ yields $G_{e}=0$. A second challenge to tracking control emerges from the possibility that, from points $\xi$ in $C_{p}$ that are nearby $D_{p}$ and times $(t, j) \in \mathcal{T}_{r}, G_{e}$ updates $e$ to $\left|e^{+}\right|=e+1$, which is far from zero (also when $e$ is arbitrarily close to zero). In fact, Figure 2 depicts a particular map $G_{e}$ as a function of $\xi$ and $r(t, j)$ when the jumps of the plant occur when $\xi=1$, that is, $D_{p}:=\{(\xi, u): \xi=1\}$, and with $G_{p}(\xi, u)=\xi+u, \kappa_{c}(y, r)=-\xi$, resulting in $G_{p}(\xi, u)=0$ when $\xi=1$. In this case, when $(t, j) \in \mathcal{T}_{r}$ (equivalently, $r(t, j)=1$ ) if $\xi=1$ then $e^{+}=0$ (i.e. the tracking error jumps to zero); however, if $\xi$ is slightly below 1 and $(t, j) \in \mathcal{T}_{r}$, then $\left|e^{+}\right|$will be close to 1 after the jump (of the reference). This "peaking phenomenon," which is due to the jump instants of plant and reference not coinciding, has also been recognized in [Brogliato et al.(2000), Leine and van de Wouw(2008), Menini and Tornambe(2001), Heemels et al.(2011), Galeani et al.(2011)] and imposes a difficulty in guaranteeing that the norm of $e$ converges to zero.

We consider tracking controllers that tackle the first challenge above while avoiding the issue of an increasing error signal in the second challenge by ensuring that jumps of the plant occur at the same instant as the jumps of the reference trajectories. For this purpose, we recast the tracking control problem as the stabilization of a closed set which embeds the time-varying reference trajectory. For the design of the tracking controllers we exploit sufficient conditions for asymptotic stability of hybrid systems in [Goebel et al.(2012)] (see also [Sanfelice and Teel(2009)] and [Sanfelice et al.(2007)]). An alternative approach for tracking control for hybrid systems based on generating the reference trajectories from an exosystem was proposed in [Robles and Sanfelice(2011)]. 


\section{PROBLEM STATEMENT}

In this paper we consider the state tracking problem for plants modeled as hybrid systems (1)-(2). In this way, a plant is denoted $\mathcal{H}_{p}$, has state $\xi \in \mathbb{R}^{n_{p}}$, input $u \in \mathbb{R}^{m_{p}}$, and output $y=\xi$. Its dynamics are given by

$$
\mathcal{H}_{p}\left\{\begin{array}{rlrl}
\dot{\xi} & =f_{p}(\xi, u) & & (\xi, u) \in C_{p} \\
\xi^{+} & \in G_{p}(\xi, u) & & (\xi, u) \in D_{p} \\
y & =h(\xi):=\xi . &
\end{array}\right.
$$

The set $C_{p} \subset \mathbb{R}^{n_{p}} \times \mathbb{R}^{m_{p}}$ is the flow set, the function $f_{p}: C \rightarrow \mathbb{R}^{n_{p}}$ is the flow map, the set $D_{p} \subset \mathbb{R}^{n_{p}} \times \mathbb{R}^{m_{p}}$ is the jump set, $G_{p}: D \rightrightarrows \mathbb{R}^{n_{p}}$ is the jump map ${ }^{\ddagger}$, and $h$ is the output map, given by the identity function. The data of the hybrid system $\mathcal{H}_{p}$ is thus given by $\left(C_{p}, f_{p}, D_{p}, G_{p}\right.$, Id).

Solutions to hybrid systems $\mathcal{H}_{p}$ are defined by pairs of hybrid arcs $\phi_{p}$ and hybrid inputs $u$ on hybrid time domains. Hybrid time domains are subsets of $\mathbb{R}_{\geq 0} \times \mathbb{N}$ given by the union of intervals of the form $\left[t_{j}, t_{j+1}\right] \times\{j\}, t_{j} \leq t_{j+1}$. More precisely, a set $E \subset \mathbb{R}_{\geq 0} \times \mathbb{N}$ is a compact hybrid time domain if

$$
E=\bigcup_{j=0}^{J-1}\left(\left[t_{j}, t_{j+1}\right] \times\{j\}\right)
$$

for some finite sequence of jump instants $0=t_{0} \leq t_{1} \leq t_{2} \ldots \leq t_{J}$. It is a hybrid time domain if for all $(T, J) \in E, E \cap([0, T] \times\{0,1, \ldots J\})$ is a compact hybrid time domain. A function $\phi_{p}: \operatorname{dom} \phi_{p} \rightarrow \mathbb{R}^{n_{p}}$ is a hybrid arc if dom $\phi_{p}$ is a hybrid time domain and, for each $j \in \mathbb{N}$, the function $t \mapsto \phi_{p}(t, j)$ is absolutely continuous on the interval $\left\{t:(t, j) \in \operatorname{dom} \phi_{p}\right\}$. A function $u: \operatorname{dom} u \rightarrow \mathbb{R}^{m_{p}}$ is a hybrid input if $\operatorname{dom} u$ is a hybrid time domain and, for each $j \in \mathbb{N}$, the function $t \mapsto u(t, j)$ is Lebesgue measurable and locally essentially bounded on the interval $\{t:(t, j) \in \operatorname{dom} u\}$. Then, a hybrid $\operatorname{arc} \phi_{p}: \operatorname{dom} \phi_{p} \rightarrow \mathbb{R}^{n_{p}}$ and a hybrid input $u: \operatorname{dom} u \rightarrow \mathbb{R}^{m_{p}}$ define a solution pair $\left(\phi_{p}, u\right)$ to the hybrid system $\mathcal{H}_{p}$ from the initial condition $\phi_{p}(0,0)$ if the following conditions hold:

$$
\left(\phi_{p}(0,0), u(0,0)\right) \in \bar{C}_{p} \cup D_{p} \text { and } \operatorname{dom} \phi_{p}=\operatorname{dom} u\left(=\operatorname{dom}\left(\phi_{p}, u\right)\right)
$$

\footnotetext{
¥The notation $\rightrightarrows$ indicates that $G_{p}$ is a set-valued map, i.e., subsets of $D$ are mapped to subsets of $\mathbb{R}^{n_{p}}$.
} 
(S1) For each $j \in \mathbb{N}$ such that $I_{j}:=\left\{t:(t, j) \in \operatorname{dom}\left(\phi_{p}, u\right)\right\}$ has nonempty interior $\operatorname{int}\left(I_{j}\right)$,

$$
\left(\phi_{p}(t, j), u(t, j)\right) \in C_{p} \text { for all } t \in \operatorname{int}\left(I_{j}\right)
$$

and, for almost all $t \in I_{j}$,

$$
\frac{d \phi_{p}}{d t}(t, j)=f_{p}\left(\phi_{p}(t, j), u(t, j)\right)
$$

(S2) For each $(t, j) \in \operatorname{dom}\left(\phi_{p}, u\right)$ such that $(t, j+1) \in \operatorname{dom}\left(\phi_{p}, u\right)$,

$$
\left(\phi_{p}(t, j), u(t, j)\right) \in D_{p}, \quad \phi_{p}(t, j+1) \in G_{p}\left(\phi_{p}(t, j), u(t, j)\right) .
$$

A solution pair $\left(\phi_{p}, u\right)$ to $\mathcal{H}_{p}$ is said to be complete if $\operatorname{dom}\left(\phi_{p}, u\right)$ is unbounded, Zeno if it is complete but the projection of $\operatorname{dom}\left(\phi_{p}, u\right)$ onto $\mathbb{R}_{\geq 0}$ is bounded, discrete if its domain is $\{0\} \times \mathbb{N}$, and maximal if there does not exist another pair $\left(\phi_{p}, u\right)^{\prime}$ such that $\left(\phi_{p}, u\right)$ is a truncation of $\left(\phi_{p}, u\right)^{\prime}$ to some proper subset of $\operatorname{dom}\left(\phi_{p}, u\right)^{\prime}$.

We consider hybrid arcs $r: \operatorname{dom} r \rightarrow \mathbb{R}^{n_{p}}$ defining reference trajectories to be tracked by the plant $\mathcal{H}_{p}$. As for the construction of hybrid time domains above, the sequence of times corresponding to the jump instants of a reference trajectory $r$ is denoted $0=t_{0}^{r} \leq t_{1}^{r} \leq t_{2}^{r} \leq \ldots$. Using the definition of $\mathcal{H}_{p}$ in (9), hybrid tracking controllers with state $\eta \in \mathbb{R}^{n_{c}}$ and data $\left(C_{c}, f_{c}, D_{c}, G_{c}, \kappa_{c}\right)$ are given by

$$
\mathcal{H}_{c}\left\{\begin{array}{rlrl}
\dot{\eta} & =f_{c}(\eta, y, r) & & (\eta, y, r) \in C_{c} \\
\eta^{+} & \in G_{c}(\eta, y, r) & & (\eta, y, r) \in D_{c} \\
u & =\kappa_{c}(\eta, y, r) . &
\end{array}\right.
$$

The input of $\mathcal{H}_{c}$ has been assigned to $(y, r)$ while its output $u$ to the input of the plant $\mathcal{H}_{p}$.

The closed-loop system (9)-(10) resulting from interconnecting $\mathcal{H}_{p}$ and $\mathcal{H}_{c}$ is denoted $\mathcal{H}_{c l}$. Its state is given by

$$
(\xi, \eta) \in \mathbb{R}^{n_{p}} \times \mathbb{R}^{n_{c}}
$$


and its dynamics by ${ }^{\S}$

$$
\begin{aligned}
& \left.\left.\begin{array}{l}
{\left[\begin{array}{c}
\dot{\xi} \\
\dot{\eta}
\end{array}\right]=\left[\begin{array}{c}
f_{p}\left(\xi, \kappa_{c}(\eta, \xi, r)\right) \\
f_{c}(\eta, \xi, r)
\end{array}\right]} \\
{\left[\begin{array}{c}
\xi^{+} \\
\eta^{+}
\end{array}\right] \in\left[\begin{array}{c}
G_{p}\left(\xi, \kappa_{c}(\eta, \xi, r)\right) \\
\eta
\end{array}\right]} \\
{\left[\begin{array}{c}
\xi^{+} \\
\eta^{+}
\end{array}\right] \in\left[\begin{array}{c}
\xi \\
\text { and }(\eta, \xi, r) \in C_{c}
\end{array}\right.} \\
{\left[\begin{array}{c}
G_{c}(\eta, \xi, r) \\
\xi^{+} \\
\eta^{+}
\end{array}\right] \in\left\{\begin{array}{l}
\left(\xi, \kappa_{c}(\eta, \xi, r)\right) \in C_{p} \\
\left(\xi, \kappa_{c}(\eta, \xi, r)\right) \in D_{p} \\
\text { and }(\eta, \xi, r) \notin D_{c}
\end{array}\right.} \\
\left.\begin{array}{c}
{\left[G_{p}\left(\xi, \kappa_{c}(\eta, \xi, r)\right)\right.} \\
\eta
\end{array}\right],\left[\begin{array}{c}
\left.\xi, \kappa_{c}(\eta, \xi, r)\right) \notin D_{p} \\
\text { and }(\eta, \xi, r) \in D_{c}
\end{array}\right. \\
G_{c}(\eta, \xi, r)
\end{array}\right]\right\} \begin{array}{l}
\left(\xi, \kappa_{c}(\eta, \xi, r)\right) \in D_{p} \\
\text { and }(\eta, \xi, r) \in D_{c}
\end{array}
\end{aligned}
$$

where, for notational simplicity, we have omitted the argument $(t, j)$ of the time-varying reference $r$. Solutions to this closed-loop system are denoted $\phi=\left(\phi_{p}, \phi_{c}\right)$ and are defined as for $\mathcal{H}_{p}$ above.

Using the above definitions, our goal is to solve the following problem:

Problem ( $\star$ ): Given a plant $\mathcal{H}_{p}$ and a complete reference trajectory $r$, design the data $\left(C_{c}, f_{c}, D_{c}, G_{c}, \kappa_{c}\right)$ of the controller $\mathcal{H}_{c}$ so that the error between every plant solution $\phi_{p}$ and the reference trajectory $r$ is bounded by a class- $\mathcal{K}$ function of the difference between their initial values, which corresponds to stability, and asymptotically converging to zero, which corresponds to attractivity.

\footnotetext{
$\bar{\S}$ For the case when conditions $\left(\xi, \kappa_{c}(\eta, \xi, r)\right) \in D_{p}$ and $(\eta, \xi, r) \in D_{c}$ hold simultaneously, the jump map can instead be defined as $\left[\begin{array}{c}G_{p}\left(\xi, \kappa_{c}(\eta, \xi, r)\right) \\ G_{c}(\eta, \xi, r)\end{array}\right]$. The definition used in (11) leads to an outer semicontinuous set-valued jump map when $G_{p}$ and $G_{c}$ are outer semicontinuous. A set-valued map $S: \mathbb{R}^{n} \rightrightarrows \mathbb{R}^{m}$ is outer semicontinuous at $x \in \mathbb{R}^{n}$ if for each sequence $\left\{x_{i}\right\}_{i=1}^{\infty}$ converging to a point $x \in \mathbb{R}^{n}$ and each sequence $y_{i} \in S\left(x_{i}\right)$ converging to a point $y$, it holds that $y \in S(x)$; see [Rockafellar and Wets(1998), Definition 5.4].
} 
The next section formally introduces the notions of stability and attractivity and the proposed approach.

\section{A CLASS OF HYBRID CONTROLLERS FOR STATE TRACKING WITH KNOWN REFERENCE TRAJECTORIES}

\subsection{Main Approach}

In smooth systems, a well-known approach is to introduce the tracking error $e=\xi-r$ and then analyze the corresponding dynamics. This approach is used for autonomous systems with prespecified time-triggered state jumps in [Pavlidis(1966)]. However, in general, as pointed out in Section 2, the flow and jump sets as well as the flow and jump maps of the error dynamics depend on $t$ and $j$ explicitly, resulting in a nonautonomous hybrid system. To address this issue, we recast Problem $(\star)$, which pertains to the stabilization of a time-varying set, as the stabilization of a closed, not necessarily bounded, time-invariant set. Inspired by the idea of treating time in time-varying systems as a state, we achieve this by embedding the given reference trajectory into an extended hybrid system model and defining a set, the tracking set, imposing conditions on the state representing tracking of the given reference trajectory. More precisely, given a reference $r: \operatorname{dom} r \rightarrow \mathbb{R}^{n_{p}}$, following (4), we define the set $\mathcal{T}_{r}$ collecting all of the points $(t, j)$ in the domain of $r$ at which $r$ jumps, that is, every point $\left(t_{j}^{r}, j\right) \in \operatorname{dom} r$ for which $\left(t_{j}^{r}, j+1\right) \in \operatorname{dom} r$. Auxiliary variables $\tau \in \mathbb{R}_{\geq 0}$ and $k \in \mathbb{N}$ are incorporated as states to parametrize the given reference trajectory $r$. In other words, $\tau$ evolves continuously according to the flow time parameter $t$, while $k$ evolves discretely according to the jump time parameter $j$ at jumps of $r$. In this setting, the set to be stabilized, called the tracking set, is given by

$$
\mathcal{A}=\left\{(\xi, \eta, \tau, k) \in \mathbb{R}^{n_{p}} \times \mathbb{R}^{n_{c}} \times \mathbb{R}_{\geq 0} \times \mathbb{N}: \xi=r(\tau, k), \eta \in \Phi\right\},
$$

for some closed set $\Phi \subset \mathbb{R}^{n_{c}}$ capturing the set of points asymptotically approached by the controller's state. For instance, for the example of Section 2, the resulting tracking set $\mathcal{A}$ with the 
proposed approach is given by

$$
\left\{(\xi, \eta, \tau, k): \xi+t_{k}^{r}=\tau \in\left[t_{k}^{r}, t_{k+1}^{r}\right],\left(t_{k}^{r}, k\right) \in(0,0) \cup \mathcal{T}_{r}\right\},
$$

where $\mathcal{T}_{r}$ is given in (4). The set in (13) is closed and unbounded in the $\tau$ and $k$ components.

The next ingredient of the approach is to guarantee, by design of the controller, that the jumps of the plant and of the reference trajectory occur simultaneously. This will be a constraint in the design of the controller, which, while it restricts the type of systems for which the tracking problem can be solved, as Section 6 illustrates, permits to solve a range of relevant hybrid tracking problems.

With a controller satisfying such a property, our approach is to recast the problem under study as a stabilization problem of the set $\mathcal{A}$ for the resulting closed-loop system, which we denote by $\mathcal{H}_{c l}^{\star}=(C, F, G, D)$. Its state is given by

$$
x:=(\xi, \eta, \tau, k) \in \mathbb{R}^{n_{p}} \times \mathbb{R}^{n_{c}} \times \mathbb{R}_{\geq 0} \times \mathbb{N}=: \mathcal{X} .
$$

The flow of $\mathcal{H}_{c l}^{\star}$ is governed by

$$
\left[\begin{array}{c}
\dot{\xi} \\
\dot{\eta} \\
\dot{\tau} \\
\dot{k}
\end{array}\right]=\left[\begin{array}{c}
f_{p}\left(\xi, \kappa_{c}(\eta, \xi, r(\tau, k))\right) \\
f_{c}(\eta, \xi, r(\tau, k)) \\
1 \\
0
\end{array}\right]=: F(\xi, \eta, \tau, k)
$$

when flow of $\mathcal{H}_{p}$, the reference, and $\mathcal{H}_{c}$ is possible, i.e.,

$$
\left(\xi, \kappa_{c}(\eta, \xi, r(\tau, k))\right) \in C_{p} \quad \text { and } \quad \tau \in\left[t_{k}^{r}, t_{k+1}^{r}\right] \quad \text { and } \quad(\eta, \xi, r(\tau, k)) \in C_{c} .
$$

Points $(\xi, \eta, \tau, k)$ satisfying these conditions define the flow set $C$ of $\mathcal{H}_{c l}^{\star}$ as follows:

$$
C:=\left\{x \in \mathcal{X}:\left(\xi, \kappa_{c}(\eta, \xi, r(\tau, k))\right) \in C_{p}, \tau \in\left[t_{k}^{r}, t_{k+1}^{r}\right],(\eta, \xi, r(\tau, k)) \in C_{c}\right\} .
$$

Jumps of $\mathcal{H}_{c l}^{\star}$ are governed by

$$
\left[\begin{array}{c}
\xi^{+} \\
\eta^{+} \\
\tau^{+} \\
k^{+}
\end{array}\right] \in\left[\begin{array}{c}
G_{p}\left(\xi, \kappa_{c}(\eta, \xi, r(\tau, k))\right) \\
\eta \\
\tau \\
k+1
\end{array}\right]=: G_{1}(\xi, \eta, \tau, k)
$$


when only the plant and the reference jump, i.e.,

$$
\left(\xi, \kappa_{c}(\eta, \xi, r(\tau, k))\right) \in D_{p} \quad \text { and } \quad(\tau, k) \in \mathcal{T}_{r} \quad \text { and } \quad(\eta, \xi, r(\tau, k)) \notin D_{c},
$$

by

$$
\left[\begin{array}{c}
\xi^{+} \\
\eta^{+} \\
\tau^{+} \\
k^{+}
\end{array}\right] \in\left[\begin{array}{c}
\xi \\
G_{c}(\eta, \xi, r(\tau, k)) \\
\tau \\
k
\end{array}\right]=: G_{2}(\xi, \eta, \tau, k)
$$

when only the controller jumps, i.e.,

$$
\left(\xi, \kappa_{c}(\eta, \xi, r(\tau, k))\right) \notin D_{p} \quad \text { and } \quad \tau \in\left[t_{k}^{r}, t_{k+1}^{r}\right) \quad \text { and } \quad(\eta, \xi, r(\tau, k)) \in D_{c}
$$

and by the union of $G_{1}$ and $G_{2}$ in (16) and (18), respectively, when $\pi$

$$
\left(\xi, \kappa_{c}(\eta, \xi, r(\tau, k))\right) \in D_{p} \quad \text { and } \quad(\tau, k) \in \mathcal{T}_{r} \quad \text { and } \quad(\eta, \xi, r(\tau, k)) \in D_{c} .
$$

Points $(\xi, \eta, \tau, k)$ satisfying these conditions define the jump set $D$ of $\mathcal{H}_{c l}^{\star}$ as follows:

$$
\begin{aligned}
D & :=D_{1} \cup D_{2}, \quad D_{1}:=\left\{x \in \mathcal{X}:\left(\xi, \kappa_{c}(\eta, \xi, r(\tau, k))\right) \in D_{p},(\tau, k) \in \mathcal{T}_{r}\right\}, \\
D_{2} & :=\left\{x \in \mathcal{X}: \tau \in\left[t_{k}^{r}, t_{k+1}^{r}\right), \quad(\eta, \xi, r(\tau, k)) \in D_{c}\right\} .
\end{aligned}
$$

The jump map $G$ of $\mathcal{H}_{c l}^{\star}$ is given by

$$
G(\xi, \eta, \tau, k):= \begin{cases}G_{1}(\xi, \eta, \tau, k) & (\xi, \eta, \tau, k) \in D_{1} \backslash D_{2} \\ G_{2}(\xi, \eta, \tau, k) & (\xi, \eta, \tau, k) \in D_{2} \backslash D_{1} \\ \left\{G_{1}(\xi, \eta, \tau, k), G_{2}(\xi, \eta, \tau, k)\right\} & (\xi, \eta, \tau, k) \in D_{1} \cap D_{2},\end{cases}
$$

where $G_{1}$ and $G_{2}$ are given in (16) and (18), respectively.

The proposed approach reduces the tracking problem to the stabilization of the tracking set $\mathcal{A}$. Then, by exploiting sufficient conditions for asymptotic stability of hybrid systems, a solution to Problem $(\star)$ can be obtained using the stability notion given next. Denoting by $\mathcal{S}_{\mathcal{H}_{c l}^{\star}}\left(\phi^{\star}(0,0)\right)$ the set of maximal solutions $\phi^{\star}$ to $\mathcal{H}_{c l}^{\star}$ from $\phi^{\star}(0,0)$, the set $\mathcal{A}$ is said to be

\footnotetext{
I Note that with a controller guaranteeing that the jumps of the plant and of the reference trajectory occur simultaneously, we have $(\eta, \xi, r(\tau, k)) \in C_{p} \Leftrightarrow \tau \in\left[t_{k}^{r}, t_{k+1}^{r}\right)$ and $\left(\xi, \kappa_{c}(\eta, \xi, r(\tau, k))\right) \in D_{p} \Leftrightarrow(\tau, k) \in \mathcal{T}_{r}$, indicating that writing the conditions involving $C_{p}, D_{p}$, or $\mathcal{T}_{r}$ is equivalent.
} 
- uniformly globally stable (UGS) if there exists $\alpha \in \mathcal{K}_{\infty}$ such that each solution $\phi^{\star} \in$ $\mathcal{S}_{\mathcal{H}_{c l}^{\star}}\left(\phi^{\star}(0,0)\right)$ satisfies $\left|\phi^{\star}(t, j)\right|_{\mathcal{A}} \leq \alpha\left(\left|\phi^{\star}(0,0)\right|_{\mathcal{A}}\right)$ for all $(t, j) \in \operatorname{dom} \phi^{\star}$;

- uniformly globally attractive (UGA) if for each $\varepsilon>0$ and $\lambda>0$ there exists $N>0$ such that, for any solution $\phi^{\star} \in \mathcal{S}_{\mathcal{H}_{c l}^{\star}}\left(\phi^{\star}(0,0)\right)$ with $\left|\phi^{\star}(0,0)\right|_{\mathcal{A}} \leq \lambda,(t, j) \in \operatorname{dom} \phi^{\star}$ and $t+j \geq N$ imply $\left|\phi^{\star}(t, j)\right|_{\mathcal{A}} \leq \varepsilon ;$

- uniformly globally asymptotically stable (UGAS) if it is both uniformly globally stable and uniformly globally attractive.

In contrast with the contributions in [Menini and Tornambe(2001), Martinelli et al.(2002), Galeani et al.(2008), Galeani et al.(2011)], where the controllers only guarantee convergence to zero of the tracking error (i.e., attractivity), the results in the following section provide conditions guaranteeing that the tracking set $\mathcal{A}$ is uniformly globally asymptotically stable.

\subsection{Characterization of Hybrid Controllers}

The following result establishes a sufficient condition for stabilization of the tracking set $\mathcal{A}$.

\section{Theorem 4.1}

(Sufficient conditions for UGAS) Given a complete reference trajectory $r: \operatorname{dom} r \rightarrow \mathbb{R}^{n_{p}}$ and associated tracking set $\mathcal{A}$ in (12), if there exists a hybrid controller $\mathcal{H}_{c}$ guaranteeing that

1. The jumps of $r$ and $\mathcal{H}_{p}$ occur simultaneously;

2. There exist a function $V: \mathbb{R}^{n_{p}} \times \mathbb{R}^{n_{c}} \times \mathbb{R}_{\geq 0} \times \mathbb{N} \rightarrow \mathbb{R}$ that is continuous on $C \cup D \cup G(D)$ and locally Lipschitz on a neighborhood of $C$, functions $\alpha_{1}, \alpha_{2} \in \mathcal{K}_{\infty}$, and continuous functions $\rho_{1}, \rho_{2}, \rho_{3} \in \mathcal{P} \mathcal{D}$ such that

(a) For all $(\xi, \eta, \tau, k) \in C \cup D \cup G(D)$

$$
\alpha_{1}\left(|(\xi, \eta, \tau, k)|_{\mathcal{A}}\right) \leq V(\xi, \eta, \tau, k) \leq \alpha_{2}\left(|(\xi, \eta, \tau, k)|_{\mathcal{A}}\right)
$$

(b) For all $(\xi, \eta, \tau, k) \in C$

$$
V^{\circ}((\xi, \eta, \tau, k), F(\xi, \eta, \tau, k)) \leq-\rho_{3}\left(|(\xi, \eta, \tau, k)|_{\mathcal{A}}\right),
$$


(c) For all $(\xi, \eta, \tau, k) \in D_{1}$ and all $g \in G_{1}(\xi, \eta, \tau, k)$

$$
V(g)-V(\xi, \eta, \tau, k) \leq-\rho_{1}\left(|(\xi, \eta, \tau, k)|_{\mathcal{A}}\right)
$$

(d) For all $(\xi, \eta, \tau, k) \in D_{2}$ and all $g \in G_{2}(\xi, \eta, \tau, k)$

$$
V(g)-V(\xi, \eta, \tau, k) \leq-\rho_{2}\left(|(\xi, \eta, \tau, k)|_{\mathcal{A}}\right)
$$

then

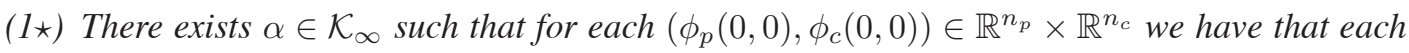
maximal solution $\phi=\left(\phi_{p}, \phi_{c}\right)$ to $\mathcal{H}_{c l}$ in (11) satisfies

$$
\left|\left(\phi_{p}(t, j)-r(t, j), \phi_{c}(t, j)\right)\right|_{\{0\} \times \Phi} \leq \alpha\left(\left|\left(\phi_{p}(0,0)-r(0,0), \phi_{c}(0,0)\right)\right|_{\{0\} \times \Phi}\right) ;
$$

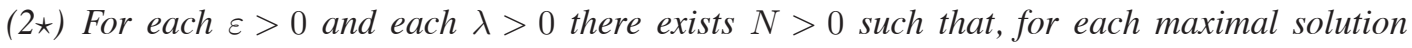
$\phi=\left(\phi_{p}, \phi_{c}\right)$ to $\mathcal{H}_{c l}$ in (11) with $\left(\phi_{p}(0,0), \phi_{c}(0,0)\right) \in \mathbb{R}^{n_{p}} \times \mathbb{R}^{n_{c}}$ such that $\mid\left(\phi_{p}(0,0)-\right.$ $\left.r(0,0), \phi_{c}(0,0)\right)\left.\right|_{\{0\} \times \Phi} \leq \lambda$ we have that

$$
(t, j) \in \operatorname{dom} \phi, t+j \geq N \quad \Rightarrow \quad\left|\left(\phi_{p}(t, j)-r(t, j), \phi_{c}(t, j)\right)\right|_{\{0\} \times \Phi} \leq \varepsilon .
$$

\section{Proof}

Let $\phi=\left(\phi_{p}, \phi_{c}\right) \in \mathcal{S}_{\mathcal{H}_{c l}}$. For each $(t, j) \in \operatorname{dom} \phi$, define the hybrid arc $\phi_{\tau}$ on dom $\phi$ such that $\phi_{\tau}(t, j)=t$. For each $i \in \mathbb{N}$, denote by $\ell_{i} \in \mathbb{N}$ the jump times $\left(t_{i}^{r}, \ell_{i}\right) \in \operatorname{dom} \phi$ at which the reference jumps, i.e., $r$ has a jump at $\left(t_{0}^{r}, \ell_{0}\right),\left(t_{1}^{r}, \ell_{1}\right),\left(t_{2}^{r}, \ell_{2}\right), \ldots \|$ Define the hybrid arc $\phi_{k}$ on $\operatorname{dom} \phi$ as

$$
\begin{array}{lll}
\phi_{k}(t, j)=0 & \forall(t, j) \in \operatorname{dom} \phi, t \in\left[0, t_{1}^{r}\right], j \in\left\{0, \ldots, \ell_{0}\right\}, \\
\phi_{k}(t, j)=\phi_{k}\left(t_{i}^{r}, \ell_{i-1}\right)+1 & \forall(t, j) \in \operatorname{dom} \phi, t \in\left[t_{i}^{r}, t_{i+1}^{r}\right], j \in\left\{\ell_{i-1}+1, \ldots, \ell_{i}\right\}, i>0 .
\end{array}
$$

Using item 1 of the assumptions, by construction,

$$
\phi^{\star}:=\left[\begin{array}{lll}
\phi^{\top}, & \phi_{\tau}, & \phi_{k}
\end{array}\right]^{\top}
$$

\footnotetext{
"Jumps of the hybrid controller are possible between $\left(t_{i}^{r}, \ell_{i}\right)$ and $\left(t_{i+1}^{r}, \ell_{i+1}\right)$, and $\ell_{i+1} \geq \ell_{i}+1$, since jumps of the plant occur when $r$ jumps, as given in item 1. 
is a maximal solution to $\mathcal{H}_{c l}^{\star}$ from $\left[\phi(0,0)^{\top}, 0,0\right]^{\top}$. Let $t(j)$ denote the smallest time $t$ such that $(t, j) \in \operatorname{dom} \phi^{\star}$, and $j(t)$ denote the smallest index $j$ such that $(t, j) \in \operatorname{dom} \phi^{\star}$. Then, following the arguments in the proof of [Goebel et al.(2012), Theorem 3.18], we have that, for each $(t, j) \in$ $\operatorname{dom} \phi^{\star}$,

$V\left(\phi^{\star}(t, j)\right)-V\left(\phi^{\star}(0,0)\right)=\int_{0}^{t} \frac{d V}{d \lambda}\left(\phi^{\star}(\lambda, j(\lambda))\right) d \lambda+\sum_{s=1}^{j}\left[V\left(\phi^{\star}(t(s), s)\right)-V\left(\phi^{\star}(t(s), s-1)\right)\right]$.

Since along flows, $\phi^{\star}(t, j) \in C$ for each $j$ and every $t \in\left(t_{j}, t_{j+1}\right)$ such that $(t, j) \in \operatorname{dom} \phi^{\star}$, using item $2 \mathrm{~b}$ and the fact that, for almost every $t \in\left(t_{j}, t_{j+1}\right), \frac{d}{d t} V\left(\phi^{*}(t, j)\right) \leq V^{\circ}\left(\phi^{*}(t, j), \dot{\phi}^{*}(t, j)\right)$ (see [Clarke(1990)] and [Sanfelice et al.(2007), Section IV.B]), we have that for each $t \in\left(t_{j}, t_{j+1}\right)$ such that $(t, j) \in \operatorname{dom} \phi^{\star}$

$$
\int_{0}^{t} \frac{d V}{d \lambda}\left(\phi^{\star}(\lambda, j(\lambda))\right) d \lambda \leq-\int_{0}^{t} \rho_{3}\left(\left|\phi^{\star}(\lambda, j(\lambda))\right|_{\mathcal{A}}\right) d \lambda
$$

Using item 1 and the construction of the jump map $G$, jumps of the plant/reference and controller are associated with different $(t, j)$ 's in $\operatorname{dom} \phi$. Define $\Gamma_{1}\left(\operatorname{dom} \phi^{\star}\right)$ and $\Gamma_{2}\left(\operatorname{dom} \phi^{\star}\right)$ as the set of such $(t, j)$ 's, respectively (in particular, $\Gamma_{1}\left(\operatorname{dom} \phi^{\star}\right)$ is the collection of points $\left(t_{i}^{r}, \ell_{i}\right)$ defined above). Using item $2 \mathrm{c}$, it follows that for each $\left(t^{\prime}, j^{\prime}\right) \in \Gamma_{1}\left(\operatorname{dom} \phi^{\star}\right)$, since only the plant and reference have a jump, we have

$$
V\left(\phi^{\star}\left(t^{\prime}, j^{\prime}+1\right)\right)-V\left(\phi^{\star}\left(t^{\prime}, j^{\prime}\right)\right) \leq-\rho_{1}\left(\left|\phi^{\star}\left(t^{\prime}, j^{\prime}\right)\right|_{\mathcal{A}}\right) .
$$

Using item $2 \mathrm{~d}$, it follows that for every $\left(t^{\prime}, j^{\prime}\right) \in \Gamma_{2}\left(\operatorname{dom} \phi^{\star}\right)$, only the controller jumps and we have

$$
V\left(\phi^{\star}\left(t^{\prime}, j^{\prime}+1\right)\right)-V\left(\phi^{\star}\left(t^{\prime}, j^{\prime}\right)\right) \leq-\rho_{2}\left(\left|\phi^{\star}\left(t^{\prime}, j^{\prime}\right)\right|_{\mathcal{A}}\right)
$$

Combining bounds (28)-(30), we obtain, for each $(t, j) \in \operatorname{dom} \phi^{\star}$,

$$
\begin{aligned}
V\left(\phi^{\star}(t, j)\right)-V\left(\phi^{\star}(0,0)\right) \leq & -\int_{0}^{t} \rho_{3}\left(\left|\phi^{\star}(\lambda, j(\lambda))\right|_{\mathcal{A}}\right) d \lambda \\
& -\sum_{\left(t^{\prime}, j^{\prime}\right) \in \Gamma_{1}\left(\operatorname{dom} \phi^{\star}\right), t^{\prime}+j^{\prime} \leq t+j} \rho_{1}\left(\left|\phi^{\star}\left(t^{\prime}, j^{\prime}\right)\right|_{\mathcal{A}}\right) \\
& -\sum_{\left(t^{\prime}, j^{\prime}\right) \in \Gamma_{2}\left(\operatorname{dom} \phi^{\star}\right), t^{\prime}+j^{\prime} \leq t+j} \rho_{2}\left(\left|\phi^{\star}\left(t^{\prime}, j^{\prime}\right)\right|_{\mathcal{A}}\right) .
\end{aligned}
$$

Due to $\rho_{1}, \rho_{2}, \rho_{3}$ taking on nonnegative values, we already have

$$
V\left(\phi^{\star}(t, j)\right) \leq V\left(\phi^{\star}(0,0)\right) \quad \forall(t, j) \in \operatorname{dom} \phi^{\star} .
$$


By the definition of hybrid arc in Section 3 and completeness of $r, \mathcal{A}$ is a closed subset of $\mathbb{R}^{n_{p}} \times \mathbb{R}^{n_{c}} \times \mathbb{R}_{\geq 0} \times \mathbb{N}$. Using the lower bound in (21), we have

$\left|\left(\phi(t, j), \phi_{\tau}(t, j), \phi_{k}(t, j)\right)\right|_{\mathcal{A}} \leq \alpha_{1}^{-1} \circ V\left(\phi^{\star}(0,0)\right)=\alpha_{1}^{-1} \circ V(\phi(0,0), 0,0) \quad \forall(t, j) \in \operatorname{dom} \phi^{\star}$,

and using the upper bound we obtain

$$
\begin{aligned}
\left|\left(\phi(t, j), \phi_{\tau}(t, j), \phi_{k}(t, j)\right)\right|_{\mathcal{A}} & \leq \alpha_{1}^{-1} \circ V(\phi(0,0), 0,0) \\
& \leq \alpha_{1}^{-1} \circ \alpha_{2}\left(\left|\left(\phi_{p}(0,0)-r(0,0), \phi_{c}(0,0)\right)\right|_{\{0\} \times \Phi}\right)
\end{aligned}
$$

for all $(t, j) \in \operatorname{dom} \phi^{\star}$. Then, $\mathcal{A}$ is uniformly globally stable for $\mathcal{H}_{c l}^{\star}$. By the definition of $\mathcal{A}$, we have that (25) holds with $\alpha:=\alpha_{1}^{-1} \circ \alpha_{2}$.

Now we show uniform global attractivity. Given $\varepsilon$ and $\lambda$ satisfying $0<\varepsilon<\lambda$, let $\varepsilon^{\prime}$ be such that $0<\varepsilon^{\prime}<\varepsilon$. Define

$$
\Omega\left(\varepsilon^{\prime}, \lambda\right):=\left\{z \in \mathbb{R}^{n_{p}} \times \mathbb{R}^{n_{c}} \times \mathbb{R}_{\geq 0} \times \mathbb{N}: \varepsilon^{\prime} \leq|z|_{\mathcal{A}} \leq \lambda\right\}
$$

and let $\lambda^{\prime}>0$ be such that $\left\{z: V(x) \leq \lambda^{\prime}\right\} \subset \Omega(0, \lambda)$. For each solution $\phi=\left(\phi_{p}, \phi_{c}\right) \in \mathcal{S}_{\mathcal{H}_{c l}}$ with $\left(\phi_{p}(0,0), \phi_{c}(0,0), 0,0\right) \in\left\{z: V(z) \leq \lambda^{\prime}\right\}$, following the construction in (27), associate a solution $\phi^{\star}$ to $\mathcal{H}_{c l}^{\star}$. It follows that each $\phi^{\star}$ satisfies (31), and due to the right-hand side of this expression being nonpositive, each $\phi^{\star}$ remains in $\Omega(0, \lambda)$. Using (21), we obtain from (31)

$$
\begin{aligned}
\alpha_{1}\left(\left|\phi^{\star}(t, j)\right|_{\mathcal{A}}\right) \leq V\left(\phi^{\star}(t, j)\right) & \leq-\rho\left(\varepsilon^{\prime}\right)\left(t+j_{1}(t, j)+j_{2}(t, j)\right)+\alpha_{2}(\lambda) \\
& \leq-\rho\left(\varepsilon^{\prime}\right)(t+j)+\alpha_{2}(\lambda)
\end{aligned}
$$

for each $(t, j) \in \operatorname{dom} \phi^{\star}$ for which $\phi^{\star}(t, j) \in \Omega\left(\varepsilon^{\prime}, \lambda\right)$, where $\rho(s):=\min \left\{\rho_{1}(s), \rho_{2}(s), \rho_{3}(s)\right\}$ for each $s \geq 0$, and, for each $i=1,2, j_{i}(t, j)$ denotes the largest $j^{\prime}$ such that $\left(t^{\prime}, j^{\prime}\right) \in \Gamma_{i}\left(\operatorname{dom} \phi^{\star}\right)$ and $t^{\prime}+j^{\prime} \leq t+j$. To arrive to (33) we have used the fact that, by construction, $j_{1}(t, j)+j_{2}(t, j)=j$. Then, it follows that

$$
t+j \geq N:=\frac{\alpha_{2}(\lambda)-\alpha_{1}(\varepsilon)}{\rho\left(\varepsilon^{\prime}\right)} \quad \Rightarrow \quad\left|\phi^{\star}(t, j)\right|_{\mathcal{A}} \leq \varepsilon
$$

Consequently, $\mathcal{A}$ is uniformly globally attractive for $\mathcal{H}_{c l}^{\star}$. By the definition of $\mathcal{A}$, we have that (26) holds. 


\section{Remark 4.2}

According to Theorem 4.1, the hybrid controller $\mathcal{H}_{c}$ has to be synthesized so that it enforces (22)(24). In particular, condition (22) depends on $f_{c}, C_{c}$ and $\kappa_{c}$; (23) depends on $\kappa_{c}$; and (24) depends on $G_{c}$ and $D_{c}$, which are all to be chosen in the design. These can be exploited in deriving constructive control design techniques for specific classes of hybrid systems. The next section introduces a synthesis procedure for a class of hybrid systems with linear maps and input-triggered jumps. Several examples in Section 6 illustrate the feasibility of the design of controllers satisfying the conditions of the theorem.

\section{Remark 4.3}

Theorem 4.1 implies that complete solutions to the closed-loop system are such that $|(\xi, \eta, \tau, k)(t, j)|_{\mathcal{A}} \rightarrow 0$ as $t+j \rightarrow \infty$, that is,

$$
|\xi(t, j)-r(\tau(t, j), k(t, j))| \rightarrow 0 \quad \text { as } t+j \rightarrow \infty .
$$

This includes all possible solutions with unconstrained initial conditions of $\tau$ and $k$, in particular, it holds for all solutions with $\tau(0,0)=k(0,0)=0$, for which $r(\tau(t, j), k(t, j))=r(t, j)$ and, consequently,

$$
|\xi(t, j)-r(t, j)| \rightarrow 0 \quad \text { as } t+j \rightarrow \infty .
$$

Note that complete solutions to $\mathcal{H}_{c l}^{\star}$ have the property that $\tau(t, j)+k(t, j)$ is unbounded as $t+j \rightarrow \infty$. Furthermore, it implies that $\xi(t, j)=r(t, j)$ on the domain of definition of solutions starting from $\xi(0,0)=r(0,0), \tau(0,0)=k(0,0)=0$, when solutions from such points exist.

Conditions $2 \mathrm{~b}-2 \mathrm{~d}$ in Theorem 4.1 can be relaxed under further conditions on the solutions to $\mathcal{H}_{c l}$. In particular, the next result following [Goebel et al.(2012), Proposition 3.24] relaxing the conditions at jumps will be useful in designing a tracking controller for a motion control system in Example 6.2. It establishes that conditions $(1 \star)$ and $(2 \star)$ in Theorem 4.1 hold if the Lyapunov function is nonincreasing during jumps, strictly decreasing during flow, and the duration of flow is sufficiently large for every solution. 


\section{Corollary 4.4}

(Special case of sufficient conditions for UGAS) Suppose that the conditions in Theorem 4.1 hold with $\rho_{1} \equiv 0$ and $\rho_{2} \equiv 0$. If for each $\lambda>0$ there exists $\gamma \in \mathcal{K}_{\infty}$ such that for every maximal solution $\phi$ to $\mathcal{H}_{c l}$ in $(11)$ with $\left|\left(\phi_{p}(0,0)-r(0,0), \phi_{c}(0,0)\right)\right|_{\{0\} \times \Phi} \leq \lambda$

$$
t+j \geq N,(t, j) \in \operatorname{dom} \phi \quad \Rightarrow \quad t \geq \gamma(N)
$$

then $(1 \star)$ and $(2 \star)$ in Theorem 4.1 hold.

\section{Proof}

The claim follows from (32) with $\rho=\rho_{3}$ and (34). In fact, continuing the proof of Theorem 4.1 from (32), we get

$$
\begin{aligned}
\alpha_{1}\left(\left|\phi^{\star}(t, j)\right|_{\mathcal{A}}\right) \leq V\left(\phi^{\star}(t, j)\right) & \leq-\rho_{3}\left(\varepsilon^{\prime}\right)\left(t+j_{1}(t, j)+j_{2}(t, j)\right)+\alpha_{2}(\lambda) \\
& \leq-\rho_{3}\left(\varepsilon^{\prime}\right) t+\alpha_{2}(\lambda) .
\end{aligned}
$$

Then, $\mid \phi^{\star}(t, j)_{\mathcal{A}} \leq \varepsilon$ holds when $t+j \geq N:=\gamma^{-1}\left(\frac{\alpha_{2}(\lambda)-\alpha_{1}(\varepsilon)}{\rho\left(\varepsilon^{\prime}\right)}\right)$.

\section{SYNTHESIS RESULTS FOR A CLASS OF HYBRID SYSTEMS WITH LINEAR MAPPINGS}

In Section 4, we have presented a characterization of tracking controllers solving Problem $(\star)$ for a general class of hybrid systems. This characterization, formalized in Theorem 4.1, requires the tracking controller to ensure that the plant and the reference jump simultaneously as well as that the tracking set is asymptotically stabilized. This section presents controller synthesis results for a particular class of hybrid systems with linear flow and jump maps in terms of constructive conditions in the form of LMIs. As in related works (see, e.g, [Bentsman and Miller(2007), Bentsman et al.(2008)]), impulsive control inputs are permitted when controlling the plants. 
Let us consider a class of plants given by the hybrid systems of the form

$$
\mathcal{H}_{p}^{\ell}\left\{\begin{array}{rlrl}
\dot{\xi} & =f_{p}(\xi, u):=A_{p} \xi+B_{p} u_{1} & & (\xi, u) \in C_{p}:=\left\{(\xi, u): u_{2}=0\right\} \\
\xi^{+} & =G_{p}(\xi, u):=E_{p} \xi+F_{p} u_{2} & & (\xi, u) \in D_{p}:=\left\{(\xi, u): u_{2} \neq 0\right\} \\
y & =h(\xi):=\xi &
\end{array}\right.
$$

where $\xi \in \mathbb{R}^{n_{p}}$ and $u_{1}, u_{2} \in \mathbb{R}^{m_{p}}$. In (37) we employ a decomposition of the control input $u=\left[\begin{array}{ll}u_{1}^{\top} & u_{2}^{\top}\end{array}\right]^{\top} \in \mathbb{R}^{2 m_{p}}$ into an impulsive control component $u_{2}$ and a non-impulsive control component $u_{1}$. Note that, compared to (9), we now consider plants with single-valued jump map $G_{p}$. In the next section, we study an example of a mechanical motion system with impulsive controls that can be modeled as in (37).

The following assumption imposes the existence of feedforward inputs $u^{r}=\left[\begin{array}{ll}u_{1}^{r \top} & u_{2}^{r \top}\end{array}\right]^{\top}$ inducing the reference $r$ to be tracked.

\section{Assumption 5.1}

The reference trajectory $r$ has a sequence of jump times $\mathcal{T}_{r}$ and there exist inputs $u_{1}^{r}$ and $u_{2}^{r}$ such that

$$
\begin{gathered}
\dot{r}=A_{p} r+B_{p} u_{1}^{r} \quad(t, j) \notin \mathcal{T}_{r} \\
r^{+}=E_{p} r+F_{p} u_{2}^{r} \quad(t, j) \in \mathcal{T}_{r},
\end{gathered}
$$

and $u_{2}^{r}(t, j) \neq 0$ if and only if $(t, j) \in \mathcal{T}_{r}$.

We propose the following family of static controllers to solve the tracking problem:

$$
\begin{aligned}
& u_{1}=u_{1}^{r}+K_{1}(\xi-r) \\
& u_{2}= \begin{cases}0 & u_{2}^{r}=0 \\
u_{2}^{r}+K_{2}(\xi-r) & u_{2}^{r} \neq 0 \text { and } u_{2}^{r}+K_{2}(\xi-r) \neq 0 \\
u_{2}^{r}+(1+\epsilon) K_{2}(\xi-r) & u_{2}^{r} \neq 0 \text { and } u_{2}^{r}+K_{2}(\xi-r)=0,\end{cases}
\end{aligned}
$$

where $\epsilon>0$ is a sufficiently small parameter and $K_{1}, K_{2}$ are controller gains to be designed. During jumps, the feedback law $u_{2}$ updates the plant state using a linear function of the state and reference. Moreover, the expression for $u_{2}$ (especially, the third line of its definition in (39)) enforces that

$$
u_{2}=0 \text { if and only if } u_{2}^{r}=0,
$$


which, when combined with the conditions in Assumption 5.1, implies that jumps of the plant always coincide with jumps of the reference trajectory. Finally, the control law (39) can be decomposed into feedforward terms $\left(u_{1}^{r}\right.$ and $\left.u_{2}^{r}\right)$ and feedback terms depending on the tracking error $(\xi-r)$.

Incorporating the auxiliary variables $(\tau, k)$ as in Section 4.1, due to the static linear controller (39), the closed-loop system $\mathcal{H}_{c l}^{\star}$ results in

$$
\mathcal{H}_{c l}^{\star, \ell}\left\{\begin{aligned}
\dot{\xi} & =A_{p} \xi+B_{p}\left(u_{1}^{r}(\tau, k)+K_{1}(\xi-r(\tau, k))\right) \\
\dot{\tau} & =1 \\
\dot{k} & =0 \\
\xi^{+} & =G_{p, c l}(\xi, \tau, k) \\
\tau^{+} & =\tau \\
k^{+} & =k+1
\end{aligned}\right\} \tau \in\left[t_{k}^{r}, t_{k+1}^{r}\right], k \in \mathbb{N}
$$

where

$G_{p, c l}(\xi, \tau, k)= \begin{cases}E_{p} \xi+F_{p}\left(u_{2}^{r}(\tau, k)+K_{2}(\xi-r(\tau, k))\right), & u_{2}^{r}(\tau, k)+K_{2}(\xi-r(\tau, k)) \neq 0 \\ E_{p} \xi+F_{p}\left(u_{2}^{r}(\tau, k)+(1+\epsilon) K_{2}(\xi-r(\tau, k))\right), & u_{2}^{r}(\tau, k)+K_{2}(\xi-r(\tau, k))=0 .\end{cases}$

We provide conditions to design the feedback gains $K_{1}, K_{2}$ and the parameter $\epsilon$ in (39) such that the tracking set $\mathcal{A}$ in (12) (which, due to the static controller considered here, is given by $\left.\mathcal{A}=\left\{(\xi, \tau, k) \in \mathbb{R}^{n_{p}} \times \mathbb{R}_{\geq 0} \times \mathbb{N}: \xi-r(\tau, k)=0\right\}\right)$ is uniformly globally asymptotically stable for the closed-loop system (41). Our first synthesis result is as follows.

\section{Theorem 5.2}

Consider a hybrid plant given by (37) and a complete hybrid reference trajectory $r$. If Assumption 5.1 holds and the following Linear Matrix Inequalities (LMIs) are feasible for real matrices $Y_{1}, Y_{2}$ and a real symmetric matrix $X$

$$
\begin{gathered}
A_{p} X+B_{p} Y_{1}+X A_{p}^{\top}+Y_{1}^{\top} B_{p}^{\top} \prec 0 \\
\left(\begin{array}{cc}
X & E_{p}^{\top}+Y_{2}^{\top} F_{p}^{\top} \\
E_{p} X+F_{p} Y_{2} & X
\end{array}\right) \succ 0
\end{gathered}
$$


then the tracking controller (39) with gains

$$
K_{1}=Y_{1} X^{-1}, \quad K_{2}=Y_{2} X^{-1}
$$

and $\epsilon>0$ such that**

$$
\left(\begin{array}{cc}
X & X E_{p}^{\top}+Y_{2}^{\top} F_{p}^{\top} \\
E_{p} X+F_{p} Y_{2} & X
\end{array}\right)+\epsilon\left(\begin{array}{cc}
0 & Y_{2}^{\top} F_{p}^{\top} \\
F_{p} Y_{2} & 0
\end{array}\right) \succ 0,
$$

guarantees that the tracking set $\mathcal{A}$ is uniformly globally asymptotically stable for the closed-loop system (41).

Proof

Note that the dynamics of the tracking error $e:=\xi-r(\tau, k)$ are given by

$$
\begin{aligned}
\dot{e} & =\left(A_{p}+B_{p} K_{1}\right) e=: f_{e}(e), & & \tau \in\left[t_{k}^{r}, t_{k+1}^{r}\right], k \in \mathbb{N}, \\
e^{+} & =\left(E_{p}+F_{p} K_{2}\right) e=: g_{e, 1}(e), & & (\tau, k) \in \mathcal{T}_{r} \text { and } u_{2}^{r}(\tau, k)+K_{2} e \neq 0, \\
e^{+} & =\left(E_{p}+F_{p}(1+\epsilon) K_{2}\right) e=: g_{e, 2}(e), & & (\tau, k) \in \mathcal{T}_{r} \text { and } u_{2}^{r}(\tau, k)+K_{2} e=0 .
\end{aligned}
$$

Consider a candidate Lyapunov function $V(e)=e^{\top} P e$ with symmetric matrix $P$ for system (45). Its evaluation along solutions of (45) gives

$$
\begin{array}{cc}
\left\langle\nabla V(e), f_{e}(e)\right\rangle=e^{\top}\left(P\left(A_{p}+B_{p} K_{1}\right)+\left(A_{p}+B_{p} K_{1}\right)^{\top} P\right) e & (\tau, k) \notin \mathcal{T}_{r}, \\
V\left(g_{e, 1}(e)\right)-V(e)=e^{\top}\left(\left(E_{p}+F_{p} K_{2}\right)^{\top} P\left(E_{p}+F_{p} K_{2}\right)-P\right) e & (\tau, k) \in \mathcal{T}_{r} \wedge u_{2}^{r}(\tau, k)+K_{2} e \neq 0, \\
V\left(g_{e, 2}(e)\right)-V(e)=e^{\top}\left(\left(E_{p}+F_{p}(1+\epsilon) K_{2}\right)^{\top} P\left(E_{p}+F_{p}(1+\epsilon) K_{2}\right)-P\right) e & (\tau, k) \in \mathcal{T}_{r} \wedge u_{2}^{r}(\tau, k)+K_{2} e=0 .
\end{array}
$$

Theorem 4.1 implies that $\mathcal{A}$ is globally asymptotically stable for (41) if the following matrix inequalities hold:

$$
\begin{gathered}
P \succ 0, \\
P\left(A_{p}+B_{p} K_{1}\right)+\left(A_{p}+B_{p} K_{1}\right)^{\top} P \prec 0,
\end{gathered}
$$

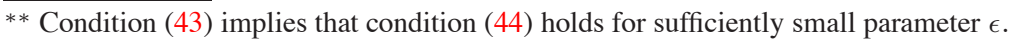




$$
\begin{gathered}
\left(E_{p}+F_{p} K_{2}\right)^{\top} P\left(E_{p}+F_{p} K_{2}\right)-P \prec 0, \\
\left(E_{p}+F_{p}(1+\epsilon) K_{2}\right)^{\top} P\left(E_{p}+F_{p}(1+\epsilon) K_{2}\right)-P \prec 0 .
\end{gathered}
$$

In fact, symmetry of the matrix $P$ implies that (21) holds with $V(e)=e^{\top} P e, \alpha_{1}(s)=\lambda_{\min }(P) s^{2}$ and $\alpha_{2}(s)=\lambda_{\max }(P) s^{2}$, where $\lambda_{\min }(P)$ and $\lambda_{\max }(P)$ are strictly positive due to (47). Moreover, (48) implies the existence of a positive definite matrix $Q_{3} \succ 0$ such that

$$
\left\langle\nabla V(e), f_{e}(e)\right\rangle \leq-e^{\top} Q_{3} e \leq-\lambda_{\min }\left(Q_{3}\right)|e|^{2}
$$

holds. Hence, (22) holds with $\rho_{3}(s)=\lambda_{\min }\left(Q_{3}\right) s^{2}$. Additionally, (49) and (50) imply the existence of a positive definite matrix $Q_{1},>0$ such that

$$
V\left(g_{e, i}(e)\right)-V(e) \leq-e^{\top} Q_{1} e \leq-\lambda_{\min }\left(Q_{1}\right)|e|^{2}, \text { for } i=1,2,
$$

from which (23) follows with $\rho_{1}(s)=\lambda_{\min }\left(Q_{1}\right) s^{2}$. We can select $\rho_{1}=\rho_{2}$, since requirement $2 \mathrm{~d}$ of Theorem 4.1 holds vacuously: the static controller (39) does not contain jumps of internal states.

Using $P \succ 0$ and applying a Schur complement to (49) gives

$$
\left(\begin{array}{cc}
P & E_{p}^{\top}+K_{2}^{\top} F_{p}^{\top} \\
E_{p}+F_{p} K_{2} & P^{-1}
\end{array}\right) \succ 0
$$

By pre- and postmultiplying this inequality by $\left[\begin{array}{cc}P^{-1} & 0 \\ 0 & I\end{array}\right]$ we obtain the equivalent expression

$$
\left(\begin{array}{cc}
P^{-1} & P^{-1} E_{p}^{\top}+P^{-1} K_{2}^{\top} F_{p}^{\top} \\
E_{p} P^{-1}+F_{p} K_{2} P^{-1} & P^{-1}
\end{array}\right) \succ 0
$$

Using the change of variables $X=P^{-1}$ and $Y_{2}=K_{2} P^{-1}$, the above inequality can be rewritten as (43) (where (43) implies that $X$ and $P$ are indeed positive definite). Observe that (49) directly implies (50) (as well as its equivalent form in (44)) for sufficiently small $\epsilon>0$, since the eigenvalues of the parameterized matrix $\left(E_{p}+F_{p}(1+\tilde{\epsilon}) K_{2}\right)^{\top} P\left(E_{p}+(1+\tilde{\epsilon}) F_{p} K_{2}\right)-P$ with $\tilde{\epsilon} \geq 0$ small are continuous in $\tilde{\epsilon}$ (see, e.g., [Kato(1995), Theorem 5.1, p. 107]).

Pre- and postmultiplying (48) by $P^{-1}$ gives equivalently

$$
P^{-1} A_{p}^{\top}+P^{-1} K_{1}^{\top} B_{p}^{\top}+A_{p} P^{-1}+B_{p} K_{1} P^{-1} \prec 0 .
$$


Applying the change of variables $X=P^{-1}$ and $Y_{1}=K_{1} P^{-1}$ gives the LMI (42). This completes the proof.

The conditions in Theorem 5.2 require the existence of a (quadratic) Lyapunov function that decays both along flows and jumps of the closed-loop system. Since this requirement may be difficult to meet in some cases, we now formulate a result that alleviates this requirement to requirements where only decay of the Lyapunov function along either flow or jumps is required together with additional requirements on the set $\mathcal{T}_{r}$ of reference jump times. These requirements are formulated by adapting the formulation of the jump time requirements of [Hespanha et al.(2008)] to our case, where multiple jumps can occur at the same time instant. This leads to the following definitions of minimum average inter-jump time and maximal average inter-jump time. Related contributions in the context of general stability (not necessarily for tracking) allowing for increase of Lyapunov functions include the work in [Michel and Hou(2011)] for dynamical systems with solutions exhibiting jumps and in [Casagrande et al.(2008)] for the stabilization of rigid bodies by means of hybrid control.

Definition 5.3 - The sequence of jump times $\left\{\left(t_{j}^{r}, j^{r}\right)\right\}$ in $\mathcal{T}_{r}$ is said to have minimal average inter-jump time $\tau>0$, if there exists an $N_{0}>0$ such that for all $(t, j) \in \operatorname{dom} r$ and all $(T, J) \in \operatorname{dom} r$ where $T+J \geq t+j$

$$
J-j \leq N_{0}+\frac{T-t}{\tau} .
$$

All jump time sequences $\left\{\left(t_{j}^{r}, j^{r}\right)\right\}$ such that (54) is satisfied are denoted by $\mathcal{S}_{\text {avg }}\left[\tau, N_{0}\right]$.

- The sequence of jump times $\left\{\left(t_{j}^{r}, j^{r}\right)\right\}$ in $\mathcal{T}_{r}$ is said to have maximal average inter-jump time $\tau>0$, if there exists an $N_{0}>0$ such that for all $(t, j) \in \operatorname{dom} r$ and all $(T, J) \in \operatorname{dom} r$ where $T+J \geq t+j$

$$
J-j \geq \frac{T-t}{\tau}-N_{0}
$$

All jump time sequences $\left\{\left(t_{j}^{r}, j^{r}\right)\right\}$ such that (55) is satisfied are denoted by $\mathcal{S}_{\text {r-avg }}[\tau]$.

As observed in [Hespanha et al.(2008)], $\mathcal{S}_{\text {avg }}[\tau, 1]$ corresponds to a minimal inter-jump time requirement with dwell-time $\tau$. For $N_{0}>1,\left\{\left(t_{j}^{r}, j^{r}\right)\right\} \in \mathcal{S}_{\text {avg }}\left[\tau, N_{0}\right]$ implies that the sequence of 
jump times satisfies an average inter-jump time requirement. Given $N_{0}>0$, condition (55) is a "reverse average inter-jump time" condition that demands jumps to occur in average at least every $\tau$ time units.

Now, we can state the following result.

\section{Theorem 5.4}

Consider a hybrid plant given by (37) and a complete hybrid reference trajectory $r$. Suppose Assumption 5.1 holds and that either of the following two conditions holds:

(A.1) the sequence of reference jump times $\left\{\left(t_{j}^{r}, j^{r}\right)\right\}$ is in $\mathcal{S}_{\text {avg }}\left[\tau, N_{0}\right]$ with $\tau \geq-\frac{d}{c}, c>0, d<0$ and $N_{0}>0$;

(A.2) the sequence of reference jump times $\left\{\left(t_{j}^{r}, j^{r}\right)\right\}$ is in $\mathcal{S}_{r-a v g}[\tau]$ with $\tau \leq-\frac{d}{c}, c<0, d>0$, $N_{0}>0$ and dom $r$ is unbounded in $t-$ direction.

If, additionally, the following LMIs are feasible for real matrices $Y_{1}, Y_{2}$ and a real symmetric matrix $X$ :

$$
\begin{gathered}
A_{p} X+B_{p} Y_{1}+X A_{p}^{\top}+Y_{1}^{\top} B_{p}^{\top}+c X \prec 0, \\
\left(\begin{array}{cc}
\exp (-d) X & X E_{p}^{\top}+Y_{2}^{\top} F_{p}^{\top} \\
E_{p} X+F_{p} Y_{2} & X
\end{array}\right) \succ 0,
\end{gathered}
$$

then the tracking controller (39) with gains $K_{1}=Y_{1} X^{-1}, K_{2}=Y_{2} X^{-1}$ and $\epsilon>0$ such that

$$
\left(\begin{array}{cc}
\exp (-d) X & X E_{p}^{\top}+Y_{2}^{\top} F_{p}^{\top} \\
E_{p} X+F_{p} Y_{2} & X
\end{array}\right)+\epsilon\left(\begin{array}{cc}
0 & Y_{2}^{\top} F_{p}^{\top} \\
F_{p} Y_{2} & 0
\end{array}\right) \succ 0,
$$

guarantees that the set $\mathcal{A}$ is a uniformly globally asymptotically stable set for the closed-loop system $(41)$.

\section{Proof}

Similar to the proof of Theorem 5.2, we prove uniform global asymptotic stability of $\mathcal{A}$ for the dynamics (41) by proving that the set $e=0$ is uniformly globally asymptotically stable for (45). Along the lines of Theorem 5.2, it can be shown that the satisfaction of the LMIs (56) and (58) guarantee that the evolution of the candidate Lyapunov function $V(e)=e^{\top} P e$, with $P=P^{\top} \succ 0$, 
along solutions of (45) satisfies

$$
\begin{aligned}
\left\langle\nabla V(e), f_{e}(e)\right\rangle & \leq-(c+\delta) V \quad(t, j) \notin \mathcal{T}_{r}, \\
V\left(g_{e, i}(e)\right) & \leq \exp (-d) V \quad(t, j) \in \mathcal{T}_{r}, \text { for } i=1,2
\end{aligned}
$$

for some $\delta>0$.

Let us now show that (59)-(60) imply that $\mathcal{A}$ is a uniformly globally asymptotically stable set of system (41) if either of the two conditions on the sequence of reference jump times $\left\{\left(t_{j}^{r}, j^{r}\right)\right\}$ posed in the theorem, i.e., condition A.1 or A.2, holds. Here, we apply analogous reasoning as presented in [Hespanha et al.(2008)], further taking into account that jumps can directly follow each other.

Let us first consider the case in which condition A.1 holds and let $T \geq 0$ be given. Since this condition requires a minimum average inter-jump time, it follows that the time domain dom $r$ is unbounded in $t$-direction, i.e., for all $T \geq 0$ there exists $J \in \mathbb{N}$ such that $(T, J) \in \operatorname{dom} r$. By construction of the controller (39) and Assumption 5.1, the time domain of (41) is equal to dom $r$, such that $e(T, J)$ can be evaluated for all $T \geq 0$ and $J$ such that $(T, J) \in \operatorname{dom} r$. From (60), (59), with $c>0$ and $d<0$, it follows that

$$
V(e(T, J)) \leq \exp (-c T-\delta T) \exp (-d J) V(e(0,0)), \text { for }(T, J) \in \operatorname{dom} r
$$

The fact that the sequence of reference jump times $\left\{\left(t_{j}^{r}, j^{r}\right)\right\}$ is in $\mathcal{S}_{\text {avg }}\left[\tau, N_{0}\right]$ implies that $J \leq$ $N_{0}+\frac{T}{\tau}$ and, hence,

$$
V(e(T, J)) \leq \exp \left(-d N_{0}\right) \exp \left(\left(-c-\frac{d}{\tau}-\delta\right) T\right) V(e(0,0)), \text { for }(T, J) \in \operatorname{dom} r,
$$

where we also used that $d<0$. Next, we use that $\tau \geq-\frac{d}{c}$, with $c>0$, implies that $-c-\frac{d}{\tau}-\delta \leq$ $-\delta<0$, which together with (62) and the fact $V(e)=e^{\top} P e$ with $P=P^{\top} \succ 0$, indeed implies that the set $e=0$ is uniformly globally asymptotically stable under the dynamics (45). Hence, $\mathcal{A}$ is uniformly globally asymptotically stable for the closed-loop system (41).

Next, let us consider the case in which condition A.2 holds and let $T \geq 0$ be given. Since dom $r$ is unbounded in $t$-direction, for all $T \geq 0$ we can choose a $J$ such that $(T, J) \in \operatorname{dom} r$, and one can evaluate $e(T, J)$ for $T \rightarrow \infty$ and appropriate $J$. 
From (60) and (59) with $c<0$ and $d>0$, it follows that

$$
V(e(T, J)) \leq \exp (-c T-\delta T) \exp (-d J) V(e(0,0)), \text { for }(T, J) \in \operatorname{dom} r
$$

The fact that the sequence of reference jump times $\left\{\left(t_{j}^{r}, j^{r}\right)\right\}$ is in $\mathcal{S}_{\mathrm{r}-\mathrm{arg}}\left[\tau, N_{0}\right]$ implies that $J \geq \frac{T}{\tau}-N_{0}$ and, hence,

$$
V(e(T, J)) \leq \exp \left(\left(-c-\frac{d}{\tau}-\delta\right) T\right) V(e(0,0)), \text { for }(T, J) \in \operatorname{dom} r
$$

where we also used that $d>0$. Next, we use that $\tau \leq-\frac{d}{c}$, with $c<0$ and $d>0$, implies that $-c-\frac{d}{\tau}-\delta \leq-\delta<0$, which together with (64) and the fact $V(e)=e^{\top} P e$, with $P=P^{\top} \succ 0$, indeed implies that the set $e=0$ is uniformly globally asymptotically stable for the system (45). Hence, $\mathcal{A}$ is uniformly globally asymptotically stable for the closed-loop system (41).

It is worth pointing out that, similarly to Theorem 5.2, condition (57) in Theorem 5.4 implies that condition (58) holds for sufficiently small parameter $\epsilon$. In this way, once condition (57) is satisfied, the design of the parameter $\epsilon$ reduces to picking a sufficiently small value such that (58) holds.

\section{EXAMPLES}

In this section, first, we illustrate our results in Section 4.2 and show that in the presented examples, a given controller solves the tracking problem. Subsequently, in three examples, the synthesis results presented in Section 5 are used to design controllers solving the tracking problem.

\section{Example 6.1}

(Tracking a periodic square wave signal) Consider the scalar hybrid plant $\mathcal{H}_{p}$

$$
\begin{array}{cl}
\dot{\xi}=-a \xi+u_{1} & \xi u_{1} \geq 0,|\xi|>0, \\
\xi^{+}=b+u_{2} & \xi u_{1} \leq 0,|\xi|>0,
\end{array}
$$


where $^{\dagger \dagger} a, b>0$, and consider the problem of tracking the square wave signal

$$
r(t, j)=(-1)^{j+1}
$$

defined for each $(t, j)$ such that $t \in\left[t_{j}^{r}, t_{j+1}^{r}\right], \quad t_{j}^{r}=j, \quad j \in \mathbb{N}$. It follows that $\mathcal{T}_{r}:=$ $\{(1,0),(2,1),(3,2), \ldots\}$. Considering static state-feedback controllers and following the approach proposed in Section 4, the goal is to solve Tracking Control Problem ( $\star$ ) with $\mathcal{A}$ given by

$\mathcal{A}=\{(\xi, \tau, k): \xi=r(\tau, k)\}=\left\{(\xi, \tau, k): \xi=(-1)^{k+1}, \tau \in\left[t_{k}^{r}, t_{k+1}^{r}\right],\left(t_{k}^{r}, k\right) \in(0,0) \cup \mathcal{T}_{r}\right\}$.

For this purpose, we consider the controller

$$
\left[\begin{array}{l}
u_{1} \\
u_{2}
\end{array}\right]=\kappa_{c}(\xi, r(\tau, k))=\left[\begin{array}{c}
a r(\tau, k) \\
-b-r(\tau, k)+\lambda(\xi-r(\tau, k))
\end{array}\right],
$$

with $\lambda \in[0,1)$. It follows that, for every initial condition $\xi(0,0)<0$, every jump of $r$ triggers a jump of the plant. In fact, if $\xi(0,0)<0$, since $u_{1}=a r(\tau, k)$, we have that $a \xi(0,0) r(0,0)>0$ and solutions initially flow. Without loss of generality, we only consider trajectories from initial conditions $\xi(0,0)<0$, since trajectories of $\xi(0,0)>0$, would directly experience a jump at $(t, j)=$ $(0,0)$, since $r(0,0)=-1$.

Flows of $\xi$ will not trigger a jump since the sign of $\xi$ remains constant. Jumps of the closed-loop system occur only when $r$ changes sign, which occurs when $(t, j) \in \mathcal{T}_{r}$.

Then, the resulting closed-loop system is given by

$$
\left.\left.\begin{array}{rl}
\dot{\xi} & =-a(\xi+r(\tau, k)) \\
\dot{\tau} & =1 \\
\dot{k} & =0
\end{array}\right\} \begin{array}{c}
a \xi r(\tau, k) \geq 0,|\xi|>0, \\
\tau \in\left[t_{k}^{r}, t_{k+1}^{r}\right], \\
\xi^{+}=-r(\tau, k)+\lambda(\xi-r(\tau, k)) \\
\tau^{+}=\tau \\
k^{+}=k+1
\end{array}\right\} \begin{gathered}
a \xi r(\tau, k) \leq 0,|\xi|>0, \\
(\tau, k) \in \mathcal{T}_{r},
\end{gathered}
$$

\footnotetext{
$\overline{\dagger^{\dagger} \text { The condition }|\xi|} \mid>0$ in the flow and jump sets of $\mathcal{H}_{p}$ removes solutions that only jump at the origin. In fact, otherwise, for the initial condition $\xi(0,0)=0$ there exists an input $u_{2}$ such that there is a solution $\phi_{p}$ satisfying $\phi_{p}(0, j)=0$ for all $j \in \mathbb{N}$.
} 
which can be written as $\mathcal{H}$ with data $(C, f, D, G)$ and captures all of the solutions to the original system with initial conditions $\xi(0,0)<0, \tau(0,0)=k(0,0)=0$. To establish asymptotic stability of $\mathcal{A}$ for (67)-(68), consider the Lyapunov function

$$
V(\xi, \tau, k)=\frac{1}{2}(\xi-r(\tau, k))^{2}
$$

for which condition (21) holds trivially, since, for each $(\xi, \tau, k) \in C \cup D \cup G(D),|(\xi, \tau, k)|_{\mathcal{A}}=$ $|\xi-r(\tau, k)| .^{\ddagger}$ For each $(\xi, \tau, k)$ satisfying $a \xi r(\tau, k) \geq 0,|\xi|>0, \tau \in\left[t_{k}^{r}, t_{k+1}^{r}\right]$, we have

$$
\langle\nabla V(\xi, \tau, k), f(\xi, \tau, k)\rangle=-a(\xi-r(\tau, k))^{2}-(\xi-r(\tau, k)) \frac{d r}{d \tau}(\tau, k)=-2 a V(\xi, \tau, k),
$$

since $\frac{\partial r}{\partial \tau}=0$ for all $\tau, k$. For each $(\xi, \tau, k)$ satisfying $a \xi r(\tau, k) \leq 0,|\xi|>0,(\tau, k) \in \mathcal{T}_{r}$, we have

$$
V(G(\xi, \tau, k))-V(\xi, \tau, k)=-\left(1-\lambda^{2}\right) V(\xi, \tau, k) .
$$

Since $a>0$ and $\lambda \in[0,1)$, Theorem 4.1 implies uniform global asymptotic stability of $\mathcal{A}$ for the closed-loop system (67)-(68) (actually, the Lyapunov function $V$ above establishes exponential stability). Figure 3(a) depicts a closed-loop system trajectory converging to the reference asymptotically, both along flows and jumps. Figure 3(b) depicts the Lyapunov function along the trajectory, which asymptotically converges to zero.

\section{Example 6.2}

(Tracking for a motion control system) Consider an inertia $M>0$ that is actuated using a force $u$, as shown in Figure 4. The position of the inertia is denoted by $\xi_{1}$ and its velocity by $\xi_{2}$. The controller force $u$ contains a Lebesgue integrable part $u_{1}$ and an impulsive part $u_{2}$ with impulses at instants $t_{i} \in \mathbb{N}$. The plant is impulsive and modeled as

$$
\dot{\xi}=\left[\begin{array}{c}
\xi_{2} \\
\frac{u_{1}}{M}
\end{array}\right] \text { when } t \neq t_{i}, \xi^{+}=\xi+\left[\begin{array}{c}
0 \\
\frac{u_{2}}{M}
\end{array}\right] \text { when } t=t_{i},
$$

\footnotetext{
¥ This follows by noting that, for the particular reference trajectory, the minimizer $x^{\prime}=\left(\xi^{\prime}, \tau^{\prime}, k^{\prime}\right)$ of $\min _{x^{\prime} \in \mathcal{A}}\left|x-x^{\prime}\right|$ for each $x \in C \cup D \cup G(D)$ must satisfy $k^{\prime}=k$. Then, the minimum distance between $(\xi, \tau, k)$ and $\mathcal{A}$ is the distance between a point and a line, which in this case is given by $|\xi-r(\tau, k)|$.
} 


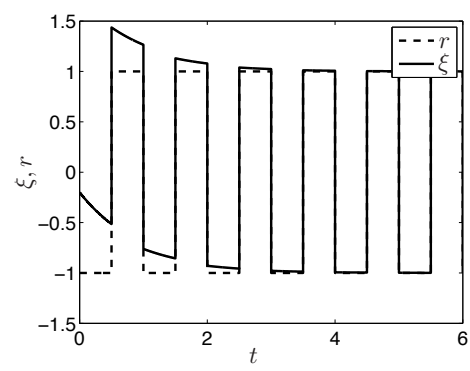

(a)

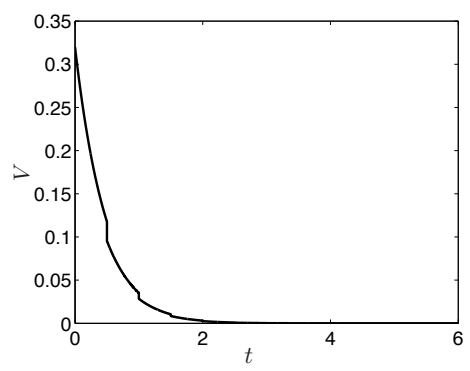

(b)

Figure 3. Reference and closed-loop system trajectory for Example 6.1. The Lyapunov function along the trajectories is also shown. Parameters: $a=b=1, \lambda=0.9$.

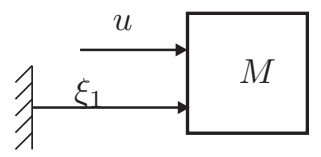

Figure 4. Schematic representation of a motion control system.

where the state $\xi$ is assumed to be completely measured. Note that the model for the plant in (69) is time dependent and, hence, different from the model in (9). The input $u$ will be designed, such that the state $\xi$ tracks a reference $r=\left[\begin{array}{l}r_{1} \\ r_{2}\end{array}\right]$, shown in Figure 5 and given by

$$
r= \begin{cases}{\left[\begin{array}{l}
t-j \\
1
\end{array}\right],} & (t, j) \in \bigcup_{k \in \mathbb{N}}[4 k, 4 k+1] \times\{4 k\} \\
{\left[\begin{array}{l}
1 \\
0
\end{array}\right],} & (t, j) \in \bigcup_{k \in \mathbb{N}}[4 k+1,4 k+2] \times\{4 k+1\} \\
{\left[\begin{array}{l}
1-t+j \\
0
\end{array}\right],} & (t, j) \in \bigcup_{k \in \mathbb{N}}[4 k+2,4 k+3] \times\{4 k+2\} \\
{\left[\begin{array}{l}
0 \\
0
\end{array}\right],} & (t, j) \in \bigcup_{k \in \mathbb{N}}[4 k+3,4 k+4] \times\{4 k+3\} .\end{cases}
$$

The component $r_{2}$ jumps at times $(t, j) \in \mathcal{T}_{r}=\bigcup_{j \in \mathbb{N}}\{(j+1, j)\}$. Such a reference trajectory can be desirable for the position of the end effector of a robot system, as it represents fast point-to-point 
motion. We propose the following control law to stabilize the set $\mathcal{A}=\{(\xi, \tau, k): \xi-r(\tau, k)=0\}$ for the given reference trajectory:

$$
\begin{aligned}
& u_{1}=-\lambda_{1}\left(\xi_{1}-r_{1}\right)-\lambda_{2}\left(\xi_{2}-r_{2}\right) \\
& u_{2}= \begin{cases}0, & (t, j) \notin \mathcal{T}_{r}, \\
M, & (t, j) \in \bigcup_{k \in \mathbb{N}}(4 k+3,4 k+2) \cup(4 k+4,4 k+3) \\
-M, & (t, j) \in \bigcup_{k \in \mathbb{N}}(4 k+1,4 k) \cup(4 k+2,4 k+1),\end{cases}
\end{aligned}
$$

where $\lambda_{1}, \lambda_{2}>0$.

Using the change of coordinates $e=\xi-r(\tau, k)$, the closed-loop system can be written as a hybrid system with state $(e, \tau, k)$ and dynamics given by

$$
\left.\begin{array}{rl}
\dot{e} & =\left[\begin{array}{cc}
0 & 1 \\
-\frac{\lambda_{1}}{M} & -\frac{\lambda_{2}}{M}
\end{array}\right] e \\
\dot{\tau} & =1 \\
\dot{k} & =0
\end{array} \quad \begin{array}{ll}
e^{+}=e \\
\tau \in\left[t_{k}^{r}, t_{k+1}^{r}\right], & \tau^{+}=\tau \\
k^{+}=k+1
\end{array}\right\}(\tau, k) \in \mathcal{T}_{r} .
$$

The feedforward signal $u_{2}$ assures that $x$ and $r$ experience equal jumps at the same time instances, such that $e$ is not affected by the jumps. Furthermore, if the initial conditions are $\xi(0,0)=$ $r(0,0), \tau(0,0)=k(0,0)=0$, then the solution satisfies $r(t, j)=\xi(t, j)$ for all $(t, j) \in \operatorname{dom} r$. Since $\lambda_{1}, \lambda_{2}>0$, the matrix $\left[\begin{array}{cc}0 & 1 \\ -\frac{\lambda_{1}}{M} & -\frac{\lambda_{2}}{M}\end{array}\right]$ is Hurwitz. Then, we can select $V(e, \tau, k)=e^{\top} P e$ with

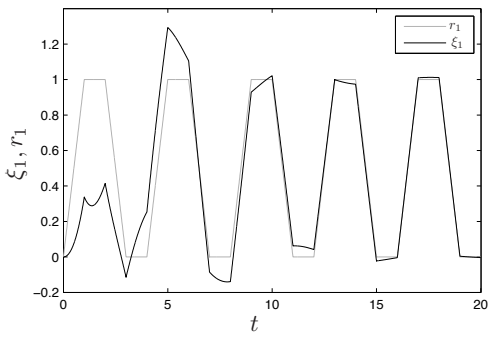

(a)

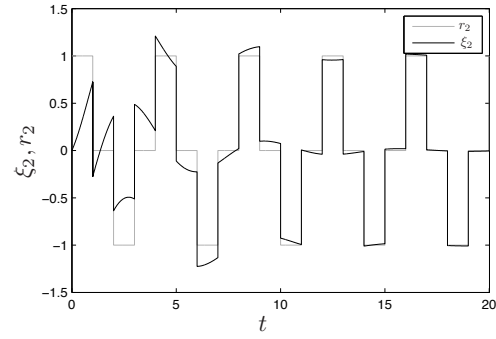

(b)

Figure 5. Reference and closed-loop trajectory for Example 6.2. Parameters: $M=1, \lambda_{1}=1$, and $\lambda_{2}=0.5$. 
$P=P^{\top} \succ 0$, such that

$$
\left\langle\nabla_{e} V(e, \tau, k), f(e)\right\rangle \leq-V(e, \tau, k)
$$

where $f(e)=\left[\begin{array}{cc}0 & 1 \\ -\frac{\lambda_{1}}{M} & -\frac{\lambda_{2}}{M}\end{array}\right] e$ follows from (72). Since $e$ is constant over jumps, we obtain

$$
V(G(e, \tau, k))-V(e, \tau, k)=0 \quad \forall(e, \tau, k),
$$

where $G(e, \tau, k)=(e, \tau, k+1)$ follows from (72). By the properties of $V$, there exist functions $\alpha_{1}, \alpha_{2}$ and $\rho$ such that both (21) and (22) are satisfied. Conditions (23) and (24) hold with $\rho_{1} \equiv 0$ and $\rho_{2} \equiv 0$. Moreover, the hybrid time domain of each solution to the closed-loop system is such that $t_{j}=j$. Then, for each element $(t, j)$ in its hybrid time domain, we have $t \geq j$ from where it follows that if $t+j \geq N$ then $t \geq \frac{N}{2}$. Hence, global uniform asymptotic stability of the set $\mathcal{A}$ for the closed-loop system (72) follows using Corollary 4.4 (note that (72) takes the form of $\mathcal{H}_{c l}^{\star}$ with sets $C_{p}$ and $C_{c}$ covering the entire state space and sets $D_{p}$ and $D_{c}$ empty). In Figure 5, a closed-loop trajectory is shown for parameters $M=1, \lambda_{1}=1$ and $\lambda_{2}=0.5$. For these system parameters, we used $P=\left[\begin{array}{cc}2.25 & 0.5 \\ 0.5 & 2\end{array}\right]$. In this example, the controller parameters are chosen to induce critically underdamped error dynamics, such that the closed-loop response shows a damped oscillation as it converges to the reference trajectory.

The next three examples illustrate the controller synthesis results presented in Section 5.

\section{Example 6.3}

(LMI-based controller synthesis without jump time restriction) Consider the system (37) with $A_{p}=$ $\left[\begin{array}{cc}0 & 1 \\ 0.1 & 0.1\end{array}\right], B_{p}=\left[\begin{array}{l}0 \\ 1\end{array}\right], E_{p}=\left[\begin{array}{cc}0.9 & 0 \\ 0 & 0.9\end{array}\right]$, and $F_{p}=\left[\begin{array}{l}0 \\ 1\end{array}\right]$. We aim to design a hybrid controller as in (39), where the controllers are synthesized using Theorem 5.2. Numerically, we find the solution $X=\left[\begin{array}{cc}7.99 & -1.84 \\ -1.84 & 9.97\end{array}\right], Y_{1}=\left[\begin{array}{ll}-9.97 & -3.33\end{array}\right]$, and $Y_{2}=\left[\begin{array}{ll}0.5 & 0.25\end{array}\right]$ to the LMIs (42), (43), which corresponds to the controller gains $K_{1}=\left[\begin{array}{ll}-1.38 & -0.59\end{array}\right]$ and $K_{2}=\left[\begin{array}{ll}0.07 & 0.04\end{array}\right]$. To illustrate the behavior of the closed-loop system, Figure 6 contains closed-loop trajectories of this system using a reference that is a solution to (38) with feedforward signal $u_{1}^{r} \equiv 0$ and $u_{2}^{r}$ given in 
(71) for $M=1$. Accurate tracking is obtained since the plant trajectory converges asymptotically to the reference. The Lyapunov function $V$ is shown to decrease both during jumps, and during flow.

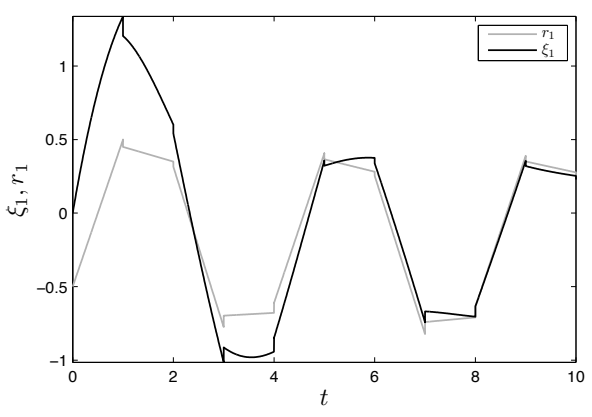

(a)

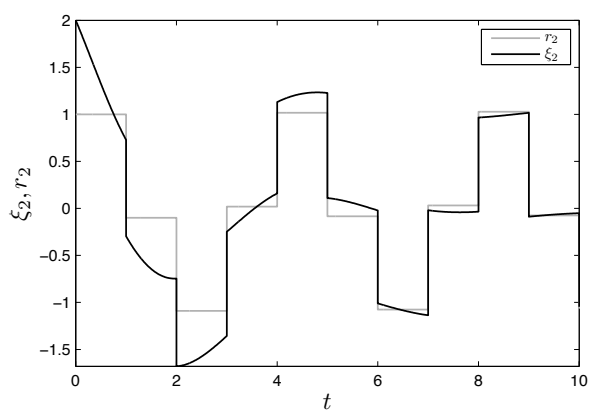

(b)

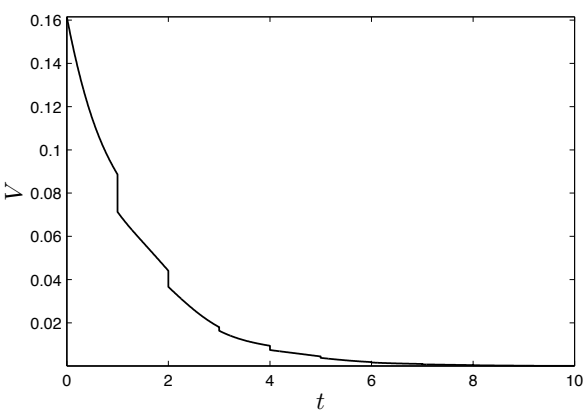

(c)

Figure 6. (a),(b) Reference and closed-loop trajectory of system (37) and controller (39) with parameters given in Example 6.3 from initial condition $r(0,0)=(-0.51)^{\top}, \xi(0,0)=(02)^{\top}$. (c) Lyapunov function evaluated along this trajectory.

\section{Example 6.4}

(LMI-based controller synthesis with a minimum average inter-jump time condition) In this example, the results in Theorem 5.4 are used to design a tracking controller for system (69) with $M=1$ and design a controller to follow reference (70), that is a solution to the hybrid system for feedforward signal $u_{1}^{r} \equiv 0$ and $u_{2}^{r}$ given in (71). Since the feedforward signal $u_{2}^{r}$ is nonzero once every continuous-time unit, we observe $\left\{\left(t_{j}^{r}, j^{r}\right)\right\} \in \mathcal{S}_{\text {avg }}[1,1]$. For $M=1$, system (69) can be written as (37) with $A_{p}=\left[\begin{array}{ll}0 & 1 \\ 0 & 0\end{array}\right], B_{p}=\left[\begin{array}{l}0 \\ 1\end{array}\right], E_{p}=\left[\begin{array}{ll}1 & 0 \\ 0 & 1\end{array}\right]$ and $F_{p}=\left[\begin{array}{l}0 \\ 1\end{array}\right]$. To use the LMI-based 


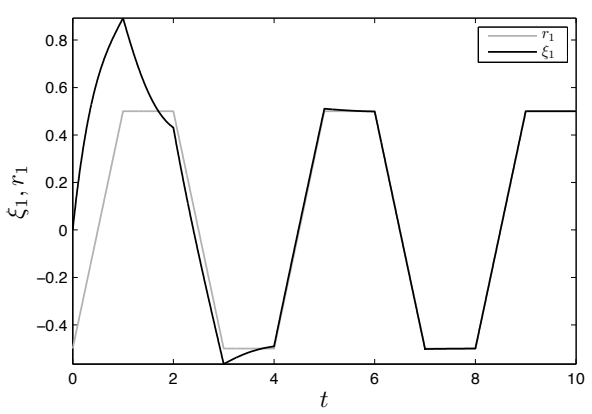

(a)

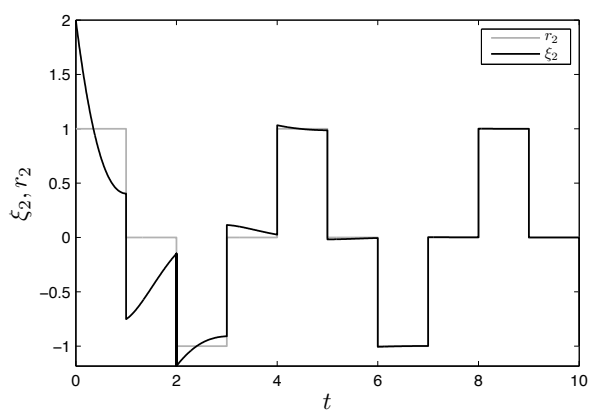

(b)

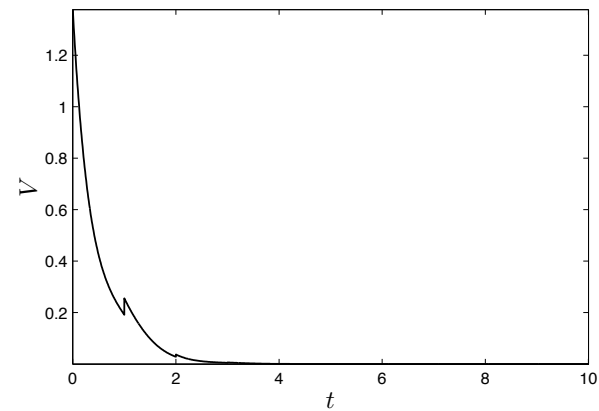

(c)

Figure 7.(a),(b) Reference and closed-loop trajectory of (69) using the synthesized controller with parameters $K_{1}=\left[\begin{array}{ll}-3.16 & -2.05\end{array}\right]$ and $K_{2}=\left[\begin{array}{ll}-0.02 & 0.25\end{array}\right]$ from initial condition $r(0,0)=\left(\begin{array}{ll}-0.5 & 1\end{array}\right)^{\top}$, $\xi(0,0)=\left(\begin{array}{ll}0 & 2\end{array}\right)^{\top}$. (c) Lyapunov function evaluated along this trajectory.

controller synthesis, we select $c=1, d=-1$, and apply a LMI solver to find solutions to the LMIs (56), (57). The solution $X=\left[\begin{array}{cc}1.05 & -0.85 \\ -0.85 & 2.42\end{array}\right], Y_{1}=\left[\begin{array}{ll}-1.57 & -2.29\end{array}\right]$, and $Y_{2}=\left[\begin{array}{ll}-0.23 & 0.61\end{array}\right]$ is obtained, yielding gains $K_{1}=\left[\begin{array}{ll}-3.16 & -2.05\end{array}\right]$ and $K_{2}=\left[\begin{array}{ll}-0.02 & 0.25\end{array}\right]$, for the controller (39).

In Figure 7, simulations are shown for the closed-loop system. In panel (a) and (b), we observe that the plant trajectory $\xi$ converges asymptotically to the reference $r$. As shown in panel (c), the Lyapunov function does increase over jumps. In fact, repetitive jumps would be unstable: the matrix $E_{p}+F_{p} K_{2}=\left[\begin{array}{cc}1 & 0 \\ -0.02 & 1.25\end{array}\right]$ has eigenvalues 1 and 1.25 . However, the unstable jumping behavior is compensated by stable behavior during flow, as depicted in panel (c). 


\section{Example 6.5}

(LMI-based controller synthesis with a maximum average inter-jump time) Consider system (37) with $A_{p}=\left[\begin{array}{cc}0 & 1 \\ 0.1 & 0.1\end{array}\right], B_{p}=\left[\begin{array}{l}0 \\ 0\end{array}\right], E_{p}=\left[\begin{array}{cc}0.5 & 0 \\ 0 & 0.5\end{array}\right]$, and $F_{p}=\left[\begin{array}{l}0 \\ 1\end{array}\right]$. Note that this system has unstable flow dynamics and allows no controller input during flow. For a reference trajectory with maximum average inter-jump time 1, i.e., $\left\{\left(t_{j}^{r}, j^{r}\right)\right\} \in \mathcal{S}_{\text {r-avg }}[1,1]$, the hybrid tracking problem can be solved. Choosing $c=-1$ and $d=1$, the LMIs (56), (57) are solved numerically to find the solution $X=\left[\begin{array}{cc}61.85 & 18.20 \\ 18.20 & 25.18\end{array}\right], Y_{2}=\left[\begin{array}{ll}2.67 & 1.00\end{array}\right]$ and arbitrary $Y_{1}$, which yields the controller gain $K_{2}=\left[\begin{array}{ll}0.04 & 0.01\end{array}\right]$. The behavior for the closed-loop system is illustrated in Figure 8 for a reference that is a solution to (37),(39) when the feedforward signal $u_{2}^{r}$ is given in (71). As shown in panel (c) of Figure 8, the Lyapunov function does increase over flow. However, this increase is compensated by decrease of the Lyapunov function at the jump instances.

\section{CONCLUSIONS}

For a class of hybrid systems given in terms of hybrid inclusions, we have stated a tracking control problem for the tracking of reference signals with jumps. The proposed technique consists of embedding the reference trajectory into an extended hybrid system model and defining a set, the tracking set, imposing conditions on the state representing tracking of the reference trajectory. Next, Lyapunov stability tools are applied to the extended system to guarantee global asymptotic stability of the tracking set. The class of controllers should ensure that the jump times of the plant coincide with those of the given reference trajectories as, otherwise, it is difficult to obtain asymptotic convergence of the tracking error to zero (relaxation of this condition is the subject of current research and may require alternative formulations of the tracking problem). Linear Matrix Inequality based synthesis techniques for a class of hybrid systems with linear flow and jump maps have been proposed and the results in the paper are illustrated by examples. 


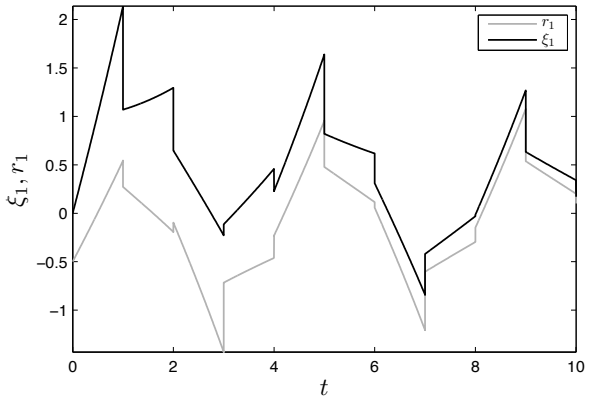

(a)

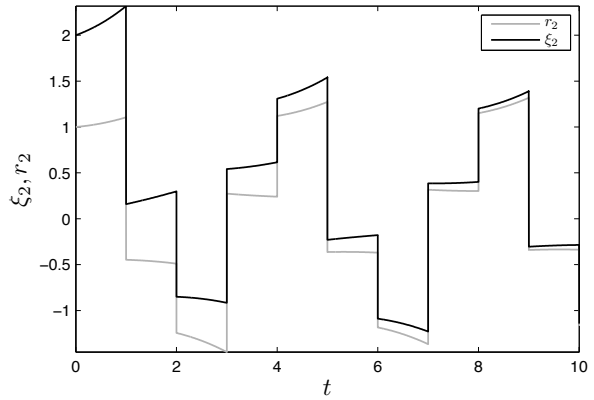

(b)

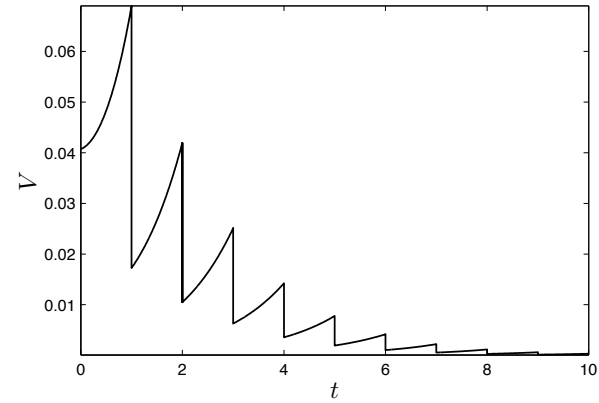

(c)

Figure 8. (a),(b) Reference and closed-loop trajectory of system (37) with controller (39) and parameters given in Section 6.5 from initial condition $r(0,0)=(-0.51)^{\top}, \xi(0,0)=\left(\begin{array}{l}0 \\ 2\end{array}\right)^{\top}$. (c) Lyapunov function evaluated along this trajectory.

\section{REFERENCES}

[Bainov and Simeonov(1989)]. Bainov, D.D., and Simeonov, P., Systems with Impulse Effect: Stability, Theory, and Applications, Ellis Horwood Limited (1989).

[Bentsman and Miller(2007)]. Bentsman, J., and Miller, B. (2007), "Dynamical systems with active singularities of elastic type: A modeling and controller synthesis framework," IEEE Transactions on Automatic Control, 52, 39-55.

[Bentsman et al.(2008)]. Bentsman, J., Miller, B., and Rubinovich, E. (2008), "Dynamical systems with active singularities: Input/state/output modeling and control," Automatica, 44, 1741-1752.

[Bourgeot and Brogliato(2005)]. Bourgeot, J.M., and Brogliato, B. (2005), "Tracking control of complementarity Lagrangian systems," Int. J. Bif. Chaos, 15, 1839-1866.

[Brogliato(1996)]. Brogliato, B., Nonsmooth Mechanics Models, Dynamics and Control, Springer (1996).

[Brogliato(2004)]. Brogliato, B. (2004), "Absolute stability and the Lagrange-Dirichlet theorem with monotone multivalued mappings," Systems \& Control Letters, 51, 343-353. 
[Brogliato et al.(1997)]. Brogliato, B., Niculescu, S., and Orhant, P. (1997), "On the control of finite-dimensional mechanical systems with unilateral constraints," IEEE Transactions on Automatic Control, 42, 200-215.

[Brogliato et al.(2000)]. Brogliato, B., Niculescu, S., and Monteiro-Marques, M. (2000), “On tracking control of a class of complementary-slackness hybrid mechanical systems," Systems and Control Letters, 39, 255-266.

[Camlibel et al.(2006)]. Camlibel, M., Pang, J.S., and Shen, J. (2006), "Lyapunov stability of complementarity and extended systems," SIAM Journal on Optimization, 17, 1056-1101.

[Casagrande et al.(2008)]. Casagrande, D., Astolfi, A., and Parisini, T. (2008), "Global asymptotic stabilization of the attitude and the angular rates of an underactuated non-symmetric rigid body," Automatica, 44, 1781-1789.

[Clarke(1990)]. Clarke, F., Optimization and Nonsmooth Analysis, SIAM's Classic in Applied Mathematics (1990).

[Devasia et al.(1996)]. Devasia, S., Chen, D., and Paden, B. (1996), "Nonlinear inversion based output tracking," IEEE Transactions on Automatic Control, 41, 930-942.

[Galeani et al.(2011)]. Galeani, S., Menini, L., and Potini, A. (2011), "Robust trajectory tracking for a class of hybrid systems: an internal model principle approach," To appear in IEEE Transactions on Automatic Control.

[Galeani et al.(2008)]. Galeani, S., Menini, L., Potini, A., and Tornambè, A. (2008), "Trajectory tracking for a particle in elliptical billiards," International Journal of Control, 81, 189-213.

[Goebel et al.(2012)]. Goebel, R., Sanfelice, R.G., and Teel, A.R., Hybrid Dynamical Systems: Modeling, Stability, and Robustness, Princeton University Press (2012).

[Goebel et al.(2009)]. Goebel, R., Sanfelice, R., and Teel, A. (2009), “Hybrid dynamical systems,” IEEEControlSystems Magazine, pp. 28-93.

[Goebel and Teel(2006)]. Goebel, R., and Teel, A. (2006), "Solutions to Hybrid Inclusions Via Set and Graphical Convergence with Stability Theory Applications," Automatica, 42, 573-587.

[Heemels et al.(2011)]. Heemels, W.P.M.H., Camlibel, M., Brogliato, B., and Schumacher, J.M. (2011), "Observerbased control of linear complementarity systems," International Journal of Robust and Nonlinear Control, 21, $1193-1218$

[Hespanha et al.(2008)]. Hespanha, J.P., Liberzon, D., and Teel, A.R. (2008), "Lyapunov conditions for input-to-state stability of impulsive systems," Automatica, 44, 2735 - 2744.

[Kato(1995)]. Kato, T., Perturbation theory for linear operators, Classics in Mathematics, Berlin Heidelberg: SpringerVerlag (1995).

[Lakshmikantham et al.(1989)]. Lakshmikantham, V., Bainov, D.D., and Simeonov, P.S., Theory of Impulsive Differential Equations, Vol. 6 of Series in Modern Applied Mathematics, Singapore: World Scientific (1989).

[Leine and van de Wouw(2008)]. Leine, R.I., and van de Wouw, N., Stability and Convergence of Mechanical Systems with Unilateral Constraints, Vol. 36 of Lecture Notes in Applied and Computational Mechanics, Berlin: Springer Verlag (2008)

[Leine and van de Wouw(2008)]. Leine, R.I., and van de Wouw, N. (2008), "Uniform convergence of monotone measure differential inclusions: with application to the control of mechanical systems with unilateral constraints," International Journal of Bifurcation and Chaos, 18, 1435-1457. 
[Lygeros et al.(2003)]. Lygeros, J., Johansson, K., Simić, S., Zhang, J., and Sastry, S.S. (2003), "Dynamical Properties of Hybrid Automata," IEEE Trans. Aut. Control, 48, 2-17.

[Martinelli et al.(2002)]. Martinelli, F., Menini, L., and Tornambe, A. (2002), "State estimation for a class of linear mechanical systems that become observable thanks to non-smooth impacts," in Proceedings of the 41st IEEE Conference on Decision and Control, Vol. 4, pp. 3608-3613.

[Menini and Tornambe(2001)]. Menini, L., and Tornambe, A. (2001), "Asymptotic tracking of periodic trajectories for a simple mechanical system subject to nonsmooth impacts," IEEE Transactions on Automatic Control, 46, 11221126.

[Michel and Hou(2011)]. Michel, A.N., and Hou, L. (2011), "Relaxation of Hypotheses in LaSalleKrasovskii-Type Invariance Results," SIAM J. Control Optim., 49, 1383-1403.

[Morarescu and Brogliato(2010)]. Morarescu, I.C., and Brogliato, B. (2010), "Trajectory Tracking Control of Multiconstraint Complementarity Lagrangian Systems," IEEE Transactions on Automatic Control, 55, 1300-1313.

[Pavlidis(1966)]. Pavlidis, T. (1966), "Stability of a class of discontinuous dynamical systems," Information and Control, 9, 298-322.

[Pereira and Silva(2004)]. Pereira, F.L., and Silva, G.N. (2004), "Lyapunov stability of measure driven impulsive systems," Differential Equations, 40, 1122-1130.

[Robles and Sanfelice(2011)]. Robles, M., and Sanfelice, R.G. (2011), "Hybrid Controllers for Tracking of Impulsive Reference Trajectories: A Hybrid Exosystem Approach," in Proc. 14th International Conference Hybrid Systems: Control and Computation, pp. 231-240.

[Rockafellar and Wets(1998)]. Rockafellar, R., and Wets, R.J.B., Variational Analysis, Springer (1998).

[Sanfelice et al.(2007)]. Sanfelice, R.G., Goebel, R., and Teel, A.R. (2007), "Invariance principles for hybrid systems with connections to detectability and asymptotic stability," IEEE Transactions on Automatic Control, 52, 22822297.

[Sanfelice and Teel(2009)]. Sanfelice, R.G., and Teel, A.R. (2009), "Asymptotic stability in hybrid systems via nested Matrosov functions," IEEE Transactions on Automatic Control, 54, 1569-1574.

[Sanfelice et al.(2007)]. Sanfelice, R., Teel, A.R., and Sepulchre, R. (2007), "A hybrid systems approach to trajectory tracking control for juggling systems," in Proc. 46th IEEE Conference on Decision and Control, pp. 5282-5287.

[Ye et al.(1998)]. Ye, H., Mitchel, A., and Hou, L. (1998), "Stability Theory for Hybrid Dynamical Systems," IEEE Trans. Aut. Control, 43, 461-474. 